\title{
Panel unit root tests in the presence of cross-1 sectional dependencies: comparison and implications for medelling
}

\author{
Citation for published version (APA):
}

Palm, F. C., Gengenbach, C., \& Urbain, J. R. Y. J. (2004). Panel unit root tests in the presence of cross-1 sectional dependencies: comparison and implications for medelling. METEOR, Maastricht University School of Business and Economics. METEOR Research Memorandum No. 039 https://doi.org/10.26481/umamet.2004039

Document status and date:

Published: 01/01/2004

DOI:

10.26481/umamet.2004039

Document Version:

Publisher's PDF, also known as Version of record

\section{Please check the document version of this publication:}

- A submitted manuscript is the version of the article upon submission and before peer-review. There can be important differences between the submitted version and the official published version of record. People interested in the research are advised to contact the author for the final version of the publication, or visit the DOI to the publisher's website.

- The final author version and the galley proof are versions of the publication after peer review.

- The final published version features the final layout of the paper including the volume, issue and page numbers.

Link to publication

\footnotetext{
General rights rights.

- You may freely distribute the URL identifying the publication in the public portal. please follow below link for the End User Agreement:

www.umlib.nl/taverne-license

Take down policy

If you believe that this document breaches copyright please contact us at:

repository@maastrichtuniversity.nl

providing details and we will investigate your claim.
}

Copyright and moral rights for the publications made accessible in the public portal are retained by the authors and/or other copyright owners and it is a condition of accessing publications that users recognise and abide by the legal requirements associated with these

- Users may download and print one copy of any publication from the public portal for the purpose of private study or research.

- You may not further distribute the material or use it for any profit-making activity or commercial gain

If the publication is distributed under the terms of Article $25 \mathrm{fa}$ of the Dutch Copyright Act, indicated by the "Taverne" license above, 
Christian Gengenbach, Franz C. Palm, Jean-Pierre Urbain

Panel Unit Root Tests in the Presence of Cross-Sectional Dependencies: Comparison and Implications for Modelling

$\mathrm{RM} / 04 / 039$

\section{METE@R}

Maastricht research school of Economics of TEchnology and ORganizations

Universiteit Maastricht

Faculty of Economics and Business Administration P.O. Box 616

NL - 6200 MD Maastricht

phone : :++31433883830

fax $\quad$ : ++31433884873 



\title{
Panel Unit Root Tests in the Presence of Cross-Sectional Dependencies: \\ COMPARISON AND IMPLICATIONS FOR MODELLING
}

\author{
Christian Gengenbach, Franz C. Palm, Jean-Pierre Urbain* \\ Department of Quantitative Economics, \\ Universiteit Maastricht, The Netherlands \\ First version: September 16, 2004 \\ This version: November 28, 2006
}

\begin{abstract}
Several panel unit root tests that account for cross section dependence using a common factor structure have been proposed in the literature recently, notably Pesaran (2005), Moon and Perron (2004) and Bai and Ng (2004a). Pesaran's (2005) cross-sectionally augmented unit root tests are designed for cases where cross-sectional dependence is due to a single factor. The Moon and Perron (2004) tests which use defactored data is similar in spirit but can account for multiple common factors. The Bai and Ng (2004a) tests allow to determine the source of non-stationarity by testing for unit roots in the common factors and the idiosyncratic factors separately.

This paper makes four contributions: (1) it compares the three testing procedures in terms of similarities and difference in the data generation process, tests, null and alternative hypotheses considered, (2) using Monte Carlo results it compares the small sample properties of the tests in models with up to two common factors, and of the panel unit root tests by Breitung and Das (2006) and Sul (2006), (3) it provides an application which illustrates the use of the tests, and (4) finally it discusses the use of the tests in modelling in general.
\end{abstract}

${ }^{*}$ Universiteit Maastricht, Faculty of Economics and Business Administration, P.O. Box 616, 6200 MD Maastricht, The Netherlands. Corresponding author: Christian Gengenbach, email: C.Gengenbach@ke.unimaas.nl. The authors would like to thank Jörg Breitung and 4 anonymous referees for helpful comments and suggestions. The usual disclaimer applies. 


\section{Introduction}

For many economic applications it is important to know whether an observed time series is stationary or non-stationary. For example, to test the validity of Purchasing Power Parity (PPP) one should examine the properties of the real exchange rates. One needs to look at the behavior of differences in real per capita output growth to test for growth convergence. Therefore, unit root tests are an important tool for econometric analysis. However, univariate unit root tests are known to lack power for samples of small or medium size. Unfortunately, for many macroeconomic variables data is available only for a small sample span. But, since studies investigating for example PPP or growth convergence are concerned with the behavior of similar data series from several countries, a natural attempt is to pool the information contained in a data panel. Indeed, that is the general idea of panel unit root tests, and they only differ in the way the information is pooled. Unfortunately, simple pooling is only valid if the units of the panel are independent of each other and sufficiently homogenous. Independence however is unlikely to hold in most applications of panel unit root tests. In cross-country analysis there might be common influences to all panel members, e.g. in PPPstudies one usually uses a common numeraire country to calculate real exchange rates.

In early approaches to panel unit root testing, the often unrealistic assumption of crosssectional independence is made. For instance, the tests proposed in Levin, Lin and Chu (2002) and Im, Pesaran and Shin (2003), denoted respectively as LLC and IPS, assume crosssectional independence, but allow for heterogeneity of the form of individual deterministic effects (constant and/or linear time trend) and heterogenous serial correlation structure of the error terms. Both methods test the same null hypothesis of non-stationarity, but differ in terms of the considered alternative and hence, in the way information is pooled. Levin, Lin and Chu (2002) study balanced panels with $N$ cross-sectional units and $T$ time series observations. They assume a homogenous first order autoregressive parameter and their test is based on the pooled t-statistic of the estimator. Im, Pesaran and Shin (2003) allow unbalanced panels with $N$ cross sectional units and $T_{i}$ time series observations for each $i=1, \ldots, N$. They propose a standardized average of individual ADF statistics to test the pooled unit root null hypothesis against a heterogenous alternative. Both methods assume cross-sectional independence among panel units except for a common time effect. In that case, the derived results remain valid if cross-sectional averages are subtracted from the data.

Attention has been drawn recently to the assumption of cross-sectional independence on which the asymptotic results of both procedures rely. Among the first to analyze the effect of cross-sectional correlation on panel unit root tests was O'Connell (1998). Using Monte Carlo simulations he shows that the LLC test severely suffers from cross-correlation in terms of increased size and reduced power. He suggests using FGLS estimation to overcome this problem. However, estimation of the error covariance matrix becomes infeasible as $N$ and $T$ grow large. Flôres, Jorion, Preumont and Szafarz (1999) use SUR estimation of the (possibly heterogenous) AR parameter, and determine critical values for their test via 
Monte Carlo simulations. Their methodology has the disadvantage that it requires extensive simulations to determine critical values and does only account for contemporaneous crosssectional correlation. In simulation studies, Banerjee, Marcellino and Osbat (2004, 2005) assess the finite sample performance of panel unit root and cointegration tests when panel members are cross-correlated or even cross-sectionally cointegrated ${ }^{1}$. Their finding is, that all methods experience size distortions when panel members are cointegrated. This means that procedures such as the LLC or IPS test would over-reject the non-stationarity null when there are common sources of non-stationarity. This is analytically confirmed by Lyhagen (2000).

Recently, panel unit root tests have been proposed model cross-sectional correlation using a common factor representation of the data, or robust methods allowing for a general form of cross-sectional dependence, e.g. Chang (2002). The purpose of this paper is to study some of the new methods which assume a factor structure and compare them in terms of modelling, assumptions and statistical properties of the test statistics. A Monte Carlo study assesses the finite sample properties of the test statistics in terms of size and power in order to compare them.

Three different newly proposed unit root tests will be considered. Pesaran (2005) suggests a cross-sectionally augmented Dickey-Fuller (CADF) test where the standard DF regressions are augmented with cross-sectional averages of lagged levels and first differences of the individual series. He also considers a cross-sectional augmented IPS (CIPS) test, which is a simple average of the individual CADF-tests. The data generating process (DGP) is a simple dynamic linear heterogenous panel data model. The error term is assumed to have an unobserved one-common-factor structure accounting for cross-sectional correlation and an idiosyncratic component.

A second type of panel unit root tests has been proposed by Moon and Perron (2004). We consider two feasible t-statistics proposed by them to test for unit roots in a dynamic panel model allowing for fixed effects. The stationary error term follows a $K$-unobservedcommon-factor model to which an idiosyncratic shock is added. The t-statistics are based on appropriately standardized pooled estimators of the first order serial correlation coefficients of the data series.

The third type of panel unit root tests has been proposed by Bai and Ng (2004a). In their "Panel Analysis of Non-stationarity in Idiosyncratic and Common Components" (PANIC) approach the space spanned by the unobserved common factors and idiosyncratic disturbances is consistently estimated without knowing whether they are stationary or integrated. Next, the number of independent stochastic trends driving the common factors is determined. Both individual and pooled individual statistics are proposed to test separately for unit roots in the unobserved common and idiosyncratic components of the data instead of the observed series. Both common and idiosyncratic components may be stationary or integrated.

These three panel unit root tests have been selected for the following reasons. First of all,

\footnotetext{
${ }^{1}$ The notation of panel cointegration tests refers to tests for cointegration between several variables of one panel member, in contrast to cointegration between panel members.
} 
the model specifications are sufficiently close to each other and some are partly nested to allow for comparison. At the same time, the test procedures differ in important ways to make it interesting to compare their properties and provide some guidelines for the empirical analysis of non-stationary panel data. Second, in all the approaches an unobserved common factor structure is assumed to explain cross-sectional correlation. Common factor structures have several advantages. Statistical estimation and testing methods, and selection procedures for the number of factors are at the disposal of the empirical researcher. The statistical properties of these procedures are in general well-understood. These method recently experienced a revival in the common features literature. Using common factors to explain cross-sectional correlation allows to deal with the curse of dimensionality problem in a natural way, which has been found to work well in empirical econometrics. Finally, common factor structures often result from theoretical considerations in economics. For instance the CAPM and the APT models used in finance are common factor models, and many intertemporal microeconomic models imply factor structures for the data.

The paper is organized as follows: In Section 2 we present the DGPs used in the three approaches mentioned above. Wherever one DGP is nested in another this will be pointed out. Also, the testing procedures used will be described in some detail. We briefly discuss which features of the three approaches will be compared. In Section 3, we present the results of an extensive simulation study which compares the three approaches to panel unit root testing for models with factor structures and two panel unit root tests proposed by Breitung and Das (2006) and by Sul (2006) which do not fully exploit factor structure. A PPP test using the described methods is presented in Section 4 as an illustrative example. Section 5 is devoted to conclusions. In particular, the implications of the findings for modeling in practice will be discussed.

\section{Testing for unit roots in panel data when cross-sectional dependencies result from unobserved common factors}

This section describes three approaches to panel unit root testing in the presence of crosssectional correlation which employ factor models. In particular, the methods proposed by Pesaran (2005), Moon and Perron (2004) and Bai and Ng (2004a) will be presented. For reasons of comparison, it also briefly describes the panel unti root tests by Breitung and Das (2006) and by Sul (2006) which assume a factor structure but do not fully exploit it.

Assuming a common factor representation, one can write an observed data series $Y_{i, t}$ as the weighted sum of (unobserved) common and idiosyncratic components. For a panel with $i=1, \ldots, N$ cross-sectional units, $t=1, \ldots, T$ time series observations and $m=1, \ldots, K$ common factors, where $K<<N$, consider the following model:

$$
Y_{i, t}=\sum_{m=1}^{K} D_{i m}(L) \eta_{m, t}+C_{i}(L) \varepsilon_{i, t}, \quad i=1, \ldots, N, t=1, \ldots, T .
$$


The common shock terms $\eta_{m, t}$ are assumed to be i.i.d. $\left(0, \sigma_{f_{m}}^{2}\right)$ variables, and the idiosyncratic errors $\varepsilon_{i, t}$ are also i.i.d. $\left(0, \sigma_{\varepsilon_{i}}^{2}\right)$. Furthermore, $\eta_{m, t}$ and $\varepsilon_{i, t}$ are assumed to be mutually independent for all $i, m, t$. The lag polynomials $D_{i m}(L)=\sum_{j=1}^{\infty} d_{i m, j} L^{j}$, where $L$ is the lag operator, describe the (dynamic) dependence of the observed data on the common factor, and $C_{i}(L)=\sum_{j=1}^{\infty} c_{i, j} L^{j}$ generate individual specific dynamics. By suitably restricting these lag polynomials, it is possible to obtain the models used by Pesaran (2005), Moon and Perron (2004) and Bai and Ng (2004a) from (1). The necessary restrictions will be mentioned explicitly in the Section 2.4 .

A note on notation: Throughout this paper, $M$ is used to denote a finite, generic constant. For a matrix $A, A>0$ denotes that $A$ is positive definite. Common factors which are denoted by $f_{t}$ are always assumed to be stationary. Common factors denoted by $F_{t}$ result from an autoregressive transformation of $f_{t} . F_{t}$ has a unit root when there is a unit root in the autoregression. Whenever we refer to nonstationary common factors, this means nonstationarity of $F_{t}$.

\subsection{Pesaran(2005): A simple dynamic panel with common factors gener- ating disturbance cross-section dependence}

For a panel of observed data with $N$ cross-sectional units and $T$ time series observations, Pesaran (2005) uses a simple dynamic linear heterogenous model

$$
Y_{i, t}=\left(1-\delta_{i}\right) \mu_{i}+\delta_{i} Y_{i, t-1}+u_{i, t}, \quad i=1, \ldots, N, \quad t=1, \ldots, T,
$$

with given initial values $Y_{i, 0}$ and a one-factor structure for the disturbance

$$
u_{i, t}=\lambda_{i} \eta_{t}+\varepsilon_{i, t}
$$

The idiosyncratic shocks, $\varepsilon_{i, t}, i=1, \ldots, N, t=1, \ldots, T$ are assumed to be independently distributed both across $i$ and $t$, have zero mean, variance $\sigma_{i}^{2}$, and finite forth-order moment. The common shock $\eta_{t}$ is serially uncorrelated with mean zero and constant variance $\sigma_{f}^{2}$, and

finite forth-order moment. Without loss of generality, $\sigma_{f}^{2}$ is set equal to one. The variables $\varepsilon_{i, t}, \lambda_{i}$ and $\eta_{t}$ are assumed to be mutually independent for all $i$ and $t$.

It is convenient to write (2) and (3) as

$$
\Delta Y_{i, t}=\alpha_{i}-\left(1-\delta_{i}\right) Y_{i, t-1}+\lambda_{i} \eta_{t}+\varepsilon_{i, t}
$$

where $\alpha_{i}=\left(1-\delta_{i}\right) \mu_{i}$ and $\Delta Y_{i, t}=Y_{i, t}-Y_{i, t-1}$. The unit root hypothesis considered by Pesaran (2005), $\delta_{i}=1$ for all $i$ is tested against the possibly heterogenous alternative $\delta_{i}<1$ for $i=1, \ldots, N_{1}, \delta_{i}=1$ for $i=N_{1}+1, \ldots, N$. Pesaran (2005) assumes that $\frac{N_{1}}{N}$, the fraction of the individual processes that is stationary, is non-zero and tends to some fixed value $\kappa$ such that $0<\kappa \leq 1$ as $N \rightarrow \infty$.

The assumptions made above imply that the composite disturbance term $u_{i, t}$ is serially 
uncorrelated. This assumption and the one common factor assumption, $K=1$, could be relaxed. In fact, Pesaran (2005) considers an example where he includes $p$ lagged values of $u_{i, t}$ in (3) to obtain a stationary $p$-th order autoregression for $u_{i, t}$. This can be inverted to yield

$$
u_{i, t}=\lambda_{i} f_{t}+e_{i, t}
$$

where $f_{t}=\Phi(L) \eta_{t}$ and $e_{i, t}=\Phi(L) \varepsilon_{i, t}$ are stationary and invertible MA processes, with $\Phi(L)^{-1}$ being the AR polynomial of $u_{i, t}$.

It is important to notice that any non-stationarity of the observations $Y_{i, t}$ in the setting considered by Pesaran (2005) is due to the presence of a unit root in the autoregressive part of (2), i.e. $\delta_{i}=1$. For the unit root null hypothesis considered by Pesaran (2005), he proposes a test based on the t-ratio of the OLS estimate $\hat{b}_{i}$ in the following cross-sectionally augmented $\mathrm{DF}(\mathrm{CADF})$ regression

$$
\Delta Y_{i, t}=a_{i}+b_{i} Y_{i, t-1}+c_{i} \bar{Y}_{t-1}+d_{i} \Delta \bar{Y}_{t}+\epsilon_{i, t}
$$

where $\bar{Y}_{t}=\frac{1}{N} \sum_{i=1}^{N} Y_{i, t}, \Delta \bar{Y}_{t}=\frac{1}{N} \sum_{i=1}^{N} \Delta Y_{i, t}$, and $\epsilon_{i, t}$ is the regression error ${ }^{2}$.

The cross-sectional averages, $\bar{Y}_{t-1}$ and $\Delta \bar{Y}_{t}$, are included into (5) as a proxy for the unobserved common factor $f_{t}$. For analytical convenience when deriving the asymptotic properties, Pesaran (2005) replaces the usual estimator for $\sigma_{i}^{2}$ in the t-value for $b_{i}$ by a slightly modified and also consistent one. He derives the asymptotic distribution of the modified tstatistic and shows that it is free of nuisance parameters as $N \rightarrow \infty$ for any fixed $T>3$, as well as for the case where $N \rightarrow \infty$ followed by $T \rightarrow \infty$.

In line with Im, Pesaran and Shin (2003), Pesaran (2005) proposes a cross-sectional augmented version of the IPS-test

$$
C I P S=\frac{1}{N} \sum_{i=1}^{N} C A D F_{i}
$$

where $C A D F_{i}$ is the cross-sectionally augmented Dickey-Fuller statistic for the $\mathrm{i}$-th crosssectional unit given by the t-ratio of $b_{i}$ in the CADF regression (5). The distribution of the CIPS statistic is shown to be non-standard even for large $N$. This is in contrast to the results obtained by Im et al. (2003) under cross-sectional independence, where a standardized average of individual ADF statistics was found to be normally distributed for sufficiently large $N$.

\footnotetext{
${ }^{2}$ Note that (5) is valid for serially uncorrelated $u_{i, t}$. For the more general case, lagged values of $\Delta Y_{i, t}$, but also of $\Delta \bar{Y}_{t}$ need to be included in the estimation.
} 


\subsection{Moon and Perron (2004): A dynamic panel model with a K common factor error term}

For a panel of observed data with $N$ cross-sectional units and $T$ time series observations, Moon and Perron (2004) model the DGP for $Y_{i, t}$ as an AR(1) process and assume, similar to Pesaran (2005), that common factors are present in the error term. They assume a $K$-factor model for the error term $u_{i, t}$

$$
\begin{aligned}
Y_{i, t} & =\left(1-\delta_{i}\right) \mu_{i}+\delta_{i} Y_{i, t-1}+u_{i, t} \\
u_{i, t} & =\lambda_{i}^{\prime} f_{t}+e_{i, t}
\end{aligned}
$$

for $i=1, \ldots, N$ and $t=1, \ldots, T$, where $f_{t}$ is a $(K \times 1)$ vector of common factors, $\lambda_{i}$ is the corresponding vector of factor loading for cross-section $i$, and $e_{i, t}$ is an idiosyncratic disturbance term.

The DGPs considered by Pesaran (2005) and Moon and Perron (2004) are identical if a single common factor is present in the composite error term. For the components of the composite error term in (8) similar assumptions are made as by Pesaran (2005). The idiosyncratic part $e_{i, t}$ follows a stationary and invertible infinite MA process, and is cross-sectionally uncorrelated, so that $e_{i, t}=\Gamma_{i}(L) \varepsilon_{i, t}$, where $\Gamma_{i}(L)=\sum_{j=0}^{\infty} \gamma_{i, j} L^{j}$ and $\varepsilon_{i, t} \sim i . i . d .(0,1)$ across $i$ and $t$. Also the common factors $f_{t}$ are assumed to have a stationary, invertible $M A(\infty)$ representation, i.e. $f_{t}=\Phi(L) \eta_{t}$. Here, $\Phi(L)=\sum_{j=0}^{\infty} \phi_{j} L^{j}$ is a $K$-dimensional lag polynomial and $\eta_{t} \sim$ i.i.d. $\left(0, I_{K}\right)$. Furthermore, the covariance matrix of $f_{t}$ is (asymptotically) positive definite. Although more than one common factor are permitted to be present in the data, some maximum number $\bar{K}(\geq K)$ is supposed to be known. Also, redundant factors, i.e. factors that asymptotically influence only a finite number of observed series, are excluded by imposing $\frac{1}{N} \sum_{i=1}^{N} \lambda_{i} \lambda_{i}^{\prime} \rightarrow^{p} \Sigma_{\lambda}>0$. Furthermore, short-run and long-run variances, $\sigma_{e_{i}}^{2}\left(=\sum_{j=0}^{\infty} \gamma_{i, j}^{2}\right)$ and $\omega_{e_{i}}^{2}\left(=\left(\sum_{j=0}^{\infty} \gamma_{i, j}\right)^{2}\right)$, as well as the one sided long-run covariance $\varphi_{e_{i}}\left(=\sum_{l=1}^{\infty} \sum_{j=0}^{\infty} \gamma_{i, j} \gamma_{i, j+l}\right)$ are supposed to exist for all idiosyncratic disturbances $e_{i, t}$. Additionally, these parameters are assumed to have non-zero cross-sectional averages, $\sigma_{e}^{2}=\frac{1}{N} \sum_{i=1}^{N} \sigma_{e_{i}}^{2}, \omega_{e}^{2}=\frac{1}{N} \sum_{i=1}^{N} \omega_{e_{i}}^{2}$ and $\varphi_{e}^{2}=\frac{1}{N} \sum_{i=1}^{N} \varphi_{e_{i}}^{2}$.

The unit root null hypothesis considered by Moon and Perron (2004) is $H_{0}: \delta_{i}=1$ for all $i=1, \ldots, N$, which is tested against the heterogenous alternative $H_{1}: \delta_{i}<1$ for some $i^{3}$. To test this hypothesis, two modified t-statistics are suggested, based on pooled estimation of the first-order serial correlation coefficient of the data. The estimation and testing procedure relies on de-factoring the data by a projection onto the space orthogonal to that spanned by the common factors. For that purpose, the matrix of factor loading $\Lambda=\left(\lambda_{1}, \ldots, \lambda_{N}\right)^{\prime}$ has to

\footnotetext{
${ }^{3}$ To analyze local power properties of their test, Moon and Perron (2004) consider the following local alternative hypothesis:

$$
\delta_{i}=1-\frac{\theta_{i}}{\sqrt{N} T}
$$

where $\theta_{i}$ is a random variable with mean $\mu_{\theta}$ on finite support $[0, \bar{M}]$. The considered null hypothesis is $H_{0}^{\prime}: \mu_{\theta}=0$, which is tested against the local alternative $H_{1}^{\prime}: \mu_{\theta}>0$.
} 
be estimated to construct a projection matrix $Q_{\Lambda}=I_{N}-\Lambda\left(\Lambda^{\prime} \Lambda\right)^{-1} \Lambda^{\prime}$.

Imposing $\delta_{i}=\delta$ for all $i$, the pooled OLS estimator, denoted as $\hat{\delta}_{\text {pooled }}$, is $T$-consistent for 1 under the unit root null, as well as under the local alternative considered by Moon and Perron (2004). The usual t-ratio to test this hypothesis has a non-standard limiting distribution, due to the persistent cross-sectional correlation introduced by the common factors. From the residuals of the pooled regression (under the null where the intercept is equal to zero)

$$
\hat{u}_{i, t}=Y_{i, t}-\hat{\delta}_{\text {pooled }} Y_{i, t-1}
$$

the matrix of factor loadings is estimated by the method of principal components ${ }^{4}$. With the estimator $\hat{\Lambda}$ one can then construct an estimator of the projection matrix denoted as $Q_{\hat{\Lambda}_{K}}$. Additionally, consistent estimates of the above defined nuisance parameters can be obtained non-parametrically from the de-factored residuals $\hat{e}=\hat{u} Q_{\hat{\Lambda}_{K}}$, where $\hat{u}=\left(\hat{u}_{1}, \ldots, \hat{u}_{N}\right)$ with $\hat{u}_{i}=\left(\hat{u}_{i, 1}, \ldots, \hat{u}_{i, T}\right)^{\prime}$. Denote the estimates as $\hat{\varphi}_{e_{i}}$ and $\hat{\omega}_{e_{i}}^{2}$, and their cross-sectional averages as $\hat{\varphi}_{e}$ and $\hat{\omega}_{e}^{2}$. Then the modified pooled estimator of $\delta$ suggested by Moon and Perron (2004) is

$$
\delta_{\text {pooled }}^{*}=\frac{\sum_{t=2}^{T} Y_{t-1}^{\prime} Q_{\hat{\Lambda}_{K}} Y_{t}-N T \hat{\varphi}_{e}}{\sum_{t=2}^{T} Y_{t-1}^{\prime} Q_{\hat{\Lambda}_{K}} Y_{t-1}},
$$

where $Y_{t}=\left(Y_{1, t}, \ldots, Y_{N, t}\right)^{\prime}$. Based on this estimator, the following two t-statistics can be used to test the pooled unit root null hypothesis,

$$
t_{a}^{*}=\frac{\sqrt{N} T\left(\hat{\delta}_{\text {pooled }}^{*}-1\right)}{\sqrt{\frac{2 \hat{\phi}_{e}^{4}}{\hat{\omega}_{e}^{4}}}}
$$

and

$$
t_{b}^{*}=\sqrt{N} T\left(\hat{\delta}_{\text {pooled }}^{*}-1\right) \sqrt{\frac{1}{N T^{2}} \sum_{t=2}^{T} Y_{t-1}^{\prime} Q_{\hat{\Lambda}_{K}} Y_{t-1}}\left(\frac{\hat{\omega}_{e}}{\hat{\phi}_{e}^{2}}\right),
$$

where $\hat{\phi}_{e}^{4}=\frac{1}{N} \sum_{i=1}^{N} \hat{\phi}_{e_{i}}^{4}, \hat{\phi}_{e_{i}}^{4}=\hat{\omega}_{e_{i}}^{4}$. Moon and Perron (2004) analyze the asymptotic behavior of the two statistics as $N \rightarrow \infty$ and $T \rightarrow \infty \operatorname{with}^{5} \liminf _{(N, T \rightarrow \infty)} \frac{\log T}{\log N}>1$. Both test statistics have a limiting standard normal distribution under the null, and diverge under the stationary alternative.

\subsection{Bai and $\mathrm{Ng}$ (2004a): A common factor model with unobserved common and idiosyncratic components of unknown order of integration.}

In contrast to Pesaran (2005) or Moon and Perron (2004), the PANIC model of Bai and $\mathrm{Ng}$ (2004a) permits the non-stationarity in a panel of observed data to come either from a

\footnotetext{
${ }^{4}$ The principal component estimator is in general not unique. Moon and Perron (2004) use the normalization $\frac{1}{T} \sum_{t=1}^{T} f_{t} f_{t}^{\prime}=I_{K}$ and re-scale the obtained estimate.

${ }^{5}$ The restriction on the relative divergence rate of $N$ and $T$ is necessary, as $f_{t}$ and $e_{i, t}$ are unobserved.
} 
common source, or from the idiosyncratic errors, or from both ${ }^{6}$. Therefore, they focus on consistent estimation of the common factors and error terms, to test the properties of these series separately.

The model Bai and $\mathrm{Ng}$ (2004a) consider describes the observed data $Y_{i, t}$ as the sum of a deterministic part, a common (stochastic) component, and the idiosyncratic error. In particular,

$$
Y_{i, t}=D_{i, t}+\lambda_{i}^{\prime} F_{t}+E_{i, t} \quad i=1, \ldots, N, t=1, \ldots, T,
$$

where as before $\lambda_{i}$ is a $(K \times 1)$ vector of factor loadings, $F_{t}$ is a $(K \times 1)$ vector of common factors ${ }^{7}$, and $E_{i, t}$ is an error term. The deterministic component $D_{i, t}$ contains either a constant $\alpha_{i}$ or a linear trend $\alpha_{i}+\beta_{i}$. As the two aforementioned approaches, Bai and Ng (2004a) consider a balanced panel with $N$ cross-sectional units and, $T$ time series observations.

The common factors are assumed to follow an $A R(1)$ process, such that

$$
F_{t}=F_{t-1}+f_{t}
$$

where $f_{t}=\Phi(L) \eta_{t}, \Phi(L)=\sum_{j=1}^{\infty} \phi_{j} L^{j}$ is a $K$-dimensional lag polynomial and $\operatorname{rank}(\Phi(1))=$ $k_{1}$. So, $F_{t}$ contains $k_{1} \leq K$ independent stochastic trends and consequently $K-k_{1}$ stationary components. The shock $\eta_{t}$ is assumed to be i.i.d. $\left(0, \Sigma_{\eta}\right)$ with finite forth-order moment. The idiosyncratic terms are allowed to be either $\mathrm{I}(0)$ and $\mathrm{I}(1)$, and are also modelled as $A R(1)$ processes

$$
E_{i, t}=\delta_{i} E_{i, t-1}+e_{i, t}
$$

where $e_{i, t}$ follows a mean zero, stationary, invertible $M A$ process, such that $e_{i, t}=\Gamma_{i}(L) \varepsilon_{i, t}$ with $\varepsilon \sim$ i.i.d. $\left(0, \sigma_{\varepsilon_{i}}^{2}\right)$. Bai and $\mathrm{Ng}(2004 \mathrm{a})$ do not assume cross-sectional independence of the idiosyncratic term ${ }^{8}$ from the outset, but impose it later to validate pooled testing. The assumption that $\Sigma_{\eta}$ is not (necessarily) a diagonal matrix is more general than the corresponding assumption in Moon and Perron (2004), where the innovations of the common factors are assumed to be uncorrelated. The short-run covariance matrix of $\Delta F_{t}$ has full rank while the long-run covariance matrix has reduced rank and hence permits cointegration among the common factors. As in Moon and Perron (2004), (asymptotically) redundant factors are ruled out.

In this setup, the goal of PANIC is to determine the number of non-stationary factors $k_{1}$, and to test for each $i=1, \ldots, N$, whether $\delta_{i}=1$. Bai and $\mathrm{Ng}$ (2004) suggest using principal components to consistently estimate the unobserved components $F_{t}$ and $E_{i, t}$. However, to derive consistent estimates even if some elements of $F_{t}$ and $E_{i, t}$ are I(1), a suitable transformation of $Y_{i, t}$ is used. In particular, if the data contains only a constant, the first

\footnotetext{
${ }^{6}$ Under the unit root null the data in the Pesaran's (2005) or Moon and Perron's (2004) model contains a common, as well as an idiosyncratic stochastic trend.

${ }^{7} \mathrm{~K}$ is assumed to be known here.

${ }^{8}$ Bai and $\mathrm{Ng}(2004 \mathrm{a})$ allow for some weak cross-sectional dependence of the shock terms driving the $e_{i, t}$. The full set of assumptions can be found in their paper.
} 
differences are employed, while in the presence of a linear trend, $Y_{i, t}$ is de-trended. So, in the former case $y_{i, t}=\Delta Y_{i, t}=Y_{i, t}-Y_{i, t-1}$, while in the latter $y_{i, t}=\Delta Y_{i, t}-\Delta \bar{Y}_{i, t}$, where $\Delta \bar{Y}_{i, t}=\frac{1}{T-1} \sum_{t=2}^{T} \Delta Y_{i, t}$. As the estimated common factors and idiosyncratic errors, denoted as $\hat{f}_{t}$ and $\hat{e}_{i, t}$ respectively, are derived applying the method of principal components to firstdifferenced or de-trended data, Bai and Ng (2004a) propose to re-accumulate them to remove the effect of possible overdifferencing. This yields

$$
\begin{aligned}
\hat{F}_{t} & =\sum_{s=2}^{t} \hat{f}_{s}, \\
\hat{E}_{i, t} & =\sum_{s=2}^{t} \hat{e}_{i, s} .
\end{aligned}
$$

These estimates are now individually tested for unit roots.

For the idiosyncratic components, Bai and Ng (2004a) suggest to compute an ADF statistic based on up to $p$ lags. Denote the t-statistic to test the unit root hypothesis for each $\hat{E}_{i, t}$ as $A D F_{\hat{E}_{i}}^{c}$ or $A D F_{\hat{E}_{i}}^{\tau}$, depending on whether a constant, or a constant and linear trend is included in the DGP. Bai and Ng (2004a) derive the limiting distributions, which are nonstandard. For the case where a constant is present in the DGP given by (13), the distribution coincides with the usual Dickey-Fuller (DF) distribution where no constant is included in the estimation. The $5 \%$ critical value is -1.95 . If the DGP in (13) contains a constant and a linear trend, the limiting distribution is proportional to the reciprocal of a Brownian bridge. Critical values for this distribution are not tabulated yet, and have to be simulated.

Both $A D F$ statistics given above do not have the advantage of a standard normal limiting distribution, as do the other panel unit root tests described so far. That is due to the fact that the panel information has only been used to consistently estimate $E_{i, t}$, but not to analyze its dynamic properties. Only if independence among the error terms is assumed, pooled testing is valid. In that case, Bai and $\mathrm{Ng}$ (2004a) propose a Fisher-type test ${ }^{9}$ as suggested in Maddala and $\mathrm{Wu}$ (1999), using the correction proposed by Choi (2001). The test statistic, denoted as $P_{\hat{E}}^{c}$ or $P_{\hat{E}}^{\tau}$ depending on the deterministic specification, is given by

$$
P_{\hat{E}}^{c}, P_{\hat{E}}^{\tau}=\frac{-2 \sum_{i=1}^{N} \log \pi_{i}-2 N}{\sqrt{4 N}},
$$

where $\pi_{i}$ is the p-value of the ADF test for the $i$-th cross-section. These two panel unit root test statistics have standard normal limiting distributions.

Depending on whether there is just one, or several common factors, Bai and Ng (2004a) suggest to use either an ADF test based on up to $p$ lags, or a rank test for $\hat{F}_{t}$. Denote the t-statistic for the unit root hypothesis as $A D F_{\hat{F}}^{c}$ when only a constant is accounted for, and as $A D F_{\hat{F}}^{\tau}$ in the linear trend case. Then, Bai and $\mathrm{Ng}$ (2004a) derive their limiting distributions,

\footnotetext{
${ }^{9}$ In principal, also an IPS-type test using a standardized average of the above described t-statistics should be possible. See also Bai and Ng (2006).
} 
which coincide with the DF distributions for the cases where only a constant, or a constant and a linear trend are included in the ADF estimation. The asymptotic 5\% critical values are -2.86 and -3.41 , respectively.

If there are $K>1$ common factors, Bai and $\mathrm{Ng}$ (2004a) suggest an iterative procedure, comparable to the Johansen trace test for cointegration to select $k_{1}$. They use demeaned or de-trended factor estimates, depending on whether (13) contains just a constant, or a constant and linear trend. Define $\tilde{F}_{t}=\hat{F}_{t}-\overline{\hat{F}}_{t}$ with $\overline{\hat{F}}_{t}=\frac{1}{T-2} \sum_{t=2}^{T} \hat{F}_{t}$ in the former case. In the latter, let $\tilde{F}_{t}$ denote the residuals from a regression of $\hat{F}_{t}$ on a constant and linear trend. Using $\tilde{F}_{t}$, the following steps describe the proposed test.

Starting with $m=K$,

1. Let $\hat{\beta}_{\perp}$ be the $m$ eigenvectors associated with the $m$ largest eigenvalues of $\frac{1}{T^{2}} \sum_{t=2}^{T} \tilde{F}_{t} \tilde{F}_{t}^{\prime}$. Let $\hat{X}_{t}=\hat{\beta}_{\perp}^{\prime} \tilde{F}_{t}$. Two statistics can be considered:

2. (a) Let $K(j)=1-\frac{j}{J+1}, j=1, \ldots, J$;

i. Let $\hat{\xi}_{t}$ be the residuals from estimating a $\operatorname{VAR}(1)$ in $\hat{X}_{t}$, and let

$$
\hat{\Sigma}_{1}=\sum_{j=1}^{J} K(j)\left(\frac{1}{T} \sum_{t=2}^{T} \hat{\xi}_{t-j} \hat{\xi}_{t}^{\prime}\right)
$$

ii. Let $\hat{\nu}_{c}(m)$ be the smallest eigenvalue of

$$
\widehat{\Phi}_{c}(m)=\frac{1}{2}\left[\sum_{t=2}^{T}\left(\hat{X}_{t} \hat{X}_{t-1}^{\prime}+\hat{X}_{t-1} \hat{X}_{t}^{\prime}\right)-T\left(\hat{\Sigma}_{1}+\hat{\Sigma}_{1}^{\prime}\right)\right]\left(\sum_{t=2}^{T} \hat{X}_{t-1} \hat{X}_{t-1}^{\prime}\right)^{-1} .
$$

iii. Denote $T\left[\hat{\nu}_{c}(m)-1\right]$ as $M Q_{c}^{c}(m)$ in the constant only case, or as $M Q_{c}^{\tau}(m)$ in the linear trend case.

(b) For $p$ fixed that does not depend on $N$ or $T$,

i. Estimate a $\operatorname{Var}(\mathrm{p})$ in $\Delta \hat{X}_{t}$ in order to obtain $\widehat{\Pi}(L)=I_{m}-\widehat{\Pi}_{1} L-\cdots-\widehat{\Pi}_{p} L^{P}$. Filter $\hat{X}_{t}$ by $\widehat{\Pi}(L)$ to get $\hat{x}_{t}=\widehat{\Pi}(L) \hat{X}_{t}$.

ii. Let $\hat{\nu}_{f}(m)$ be the smallest eigenvalue of

$$
\widehat{\Phi}_{f}(m)=\frac{1}{2}\left[\sum_{t=2}^{T}\left(\hat{x}_{t} \hat{x}_{t-1}^{\prime}+\hat{x}_{t-1} \hat{x}_{t}^{\prime}\right)\right]\left(\sum_{t=2}^{T} \hat{x}_{t-1} \hat{x}_{t-1}^{\prime}\right)^{-1} .
$$

iii. Denote $T\left[\hat{\nu}_{f}(m)-1\right]$ as $M Q_{f}^{c}(m)$ in the constant only case, or as $M Q_{f}^{\tau}(m)$ in the linear trend case.

3. If $H_{0}: k_{1}=m$ is rejected, set $m=m-1$ and return to Step 1 . Otherwise, set $\hat{k}_{1}=m$ and stop.

For the $M Q_{c}^{c, \tau}$ and $M Q_{f}^{c, \tau}$ statistics described above, Bai and $\mathrm{Ng}$ (2004a) derive limiting distributions, which are again non-standard, and they provide $1 \%, 5 \%$, and $10 \%$ critical values for all four statistics and for various values of $m$. 
The PANIC procedure has the advantage that the estimated common factors and idiosyncratic components are consistent whether they are stationarity or non-stationarity. This is due to the practice of estimating the unobserved components from the first-differenced (or de-trended) data, and re-accumulating the estimates to remove the effect of possible overdifferencing if the factors or errors are stationary. Hence, the obtained estimates could also be used for stationarity tests, which is discussed in Bai and Ng (2004b).

\subsection{Alternative panel unit root tests in the presence of cross-sectional de- pendencies}

The three approaches to panel unit root testing presented in the previous sections explicitly account for the common factors employed to model the cross-sectional dependence in the data by using methods that require large $N$ to be valid. In this section we introduce alternative panel unit root tests which do not necessarily exploit the common factor structure, and could provide alternatives to the aforementioned tests in small $N$ panels. In particular, we will consider two test statistics proposed by Breitung and Das (2006) and the tests proposed by Sul (2006).

\section{Breitung and Das (2006)}

Breitung and Das (2006) study the behaviour of several panel unit root tests when crosssectional dependence in the data is present in the form of a common factor. The DGP they employ is similar to that of Bai and Ng (2004a) presented in Section 2.3 Equations (13) to (15). However, Breitung and Das (2006) focus on the special case where (14) can is replaced by

$$
F_{t}=\rho F_{t-1}+f_{t}
$$

with the scalar first order autoregressive parameter $|\rho| \leq 1$. They consider test statistics on the "reduced form" regression equation below, which is obtained when $\delta_{i}=\delta$ for all $i$ and $\rho=\delta$ :

$$
\Delta Y_{t}=\phi Y_{t-1}+u_{t}
$$

where $\Delta Y_{t}=\left(\Delta Y_{1, t}, \ldots, \Delta Y_{N, t}\right), Y_{t-1}=\left(Y_{1, t-1}, \ldots, Y_{N, t-1}\right), u_{t}=\left(u_{1, t}, \ldots, u_{N, t}\right)$ with $u_{i, t}=$ $\lambda_{i} f_{t}+e_{i, t}$ and $\phi=(\delta-1)$. The deterministic component in (13) has been assumed to be zero in this case. Breitung and Das (2006) particularly consider a robust OLS t-statistic, $t_{r o b}$ and a GLS t-statistic $t_{\text {gls }}$ to test for the unit root null hypothesis $\phi=0$ against the homogenous alternative $\phi<0$. The robust OLS statistic is now given by

$$
t_{r o b}=\frac{\sum_{t=1}^{T} Y_{t-1}^{\prime} \Delta Y_{t}}{\sum_{t=1}^{T} Y_{t-1}^{\prime} \hat{\Omega} Y_{t-1}},
$$


with $\hat{\Omega}=\sum_{t=1}^{T} \hat{u}_{t} \hat{u}_{t}^{\prime}$ where $\hat{u}_{t}=\Delta Y_{t}-\hat{\phi} Y_{t-1}$ are the OLS residuals. The GLS statistic, $t_{g l s}$, is given by

$$
t_{g l s}=\frac{\sum_{t=1}^{T} Y_{t-1}^{\prime} \hat{\Omega}^{-1} \Delta Y_{t}}{\sum_{t=1}^{T} Y_{t-1}^{\prime} \hat{\Omega}^{-1} Y_{t-1}}
$$

Note that this statistic can only be computed for $T>N$, as otherwise $\hat{\Omega}$ is singular. Also, if a common factor structure is assumed for the data, one could exploit this in for the GLS statistic by taking the factor structure into account when estimating the covariance matrix $\Omega$. For the static factor model with orthonormal factors, $\Omega=\Lambda \Lambda^{\prime}+\Sigma$, where $\Lambda$ is the $N \times k$ matrix of factor loadings and $\Sigma$ is the covariance matrix of the idiosyncratic innovations. Estimates of $\Lambda$ and $\Sigma$ can be obtained using a principal component approach as in Bai and $\mathrm{Ng}$ (2004a) or Moon and Perron (2004). If there is higher order serial correlation present in the residuals, a Newey-West type estimator for $\Omega$ can be employed, or an ADF regression estimated in the first step.

Breitung and Das (2006) consider 3 cases in their analysis, where the reduced form (19) is misspecified in cases 2 and 3, namely an I(1) common factor combined with I(1) idiosyncratic components, an I(1) common factor and I(0) idiosyncratic components (cross-member cointegration) and the case where a unit root is present in the idiosyncratic component but the common factor is $\mathrm{I}(0)$. If $\frac{N^{3}}{T} \rightarrow 0, t_{\text {gls }}$ is asymptotically normally distributed in the first and third case, while it diverges in the second case. $t_{r o b}$ converges to a Dickey-Fuller distribution in the first case if there is a single common factor. It is equivalent to an ADF test on the first principal component of $Y_{t}$ in that case. In the other cases, the test is not valid.

\section{Sul (2006)}

Sul (2006) proposes to use recursive mean adjustment for panel unit root tests to increase their power. Similar to Moon and Perron (2004), Sul (2006) models cross-sectional dependence by employing a common factor structure for the error term. The DGP is similar to that given in Equations (7) and (8). To account for the cross-sectional dependence, Sul (2006) suggests a (feasible) GLS statistic to test for the unit root null hypothesis $\delta_{i}=1$ for all $i$ against the heterogenous alternative $\delta_{i}<1$ for some $i$ in

$$
Y_{i, t}=\left(1-\delta_{i}\right) \mu_{i}+\delta_{i} Y_{i, t-1}+u_{i, t}
$$

The test procedure follows multiple steps, where the regression can be augmented by lagged first differences of $Y_{i, t}$ to account for higher order serial correlation in the residuals:

1. Run the following regression for each unit individually

$$
Y_{i, t}-c_{i, t}=\delta_{i}\left(Y_{i, t-1}-c_{i, t-1}\right)+\sum_{j=1}^{p_{i}} \varphi_{i j} \Delta Y_{i, t-j}+\epsilon_{i, t},
$$

where $c_{i, t}=(t-1)^{-1} \sum_{s=1}^{t-1} Y_{i, s}$ is the recursive mean, to obtain the LS estimator $\hat{\delta}_{i}$. 
2. If $\hat{\delta}_{i}>1$ set $\hat{\delta}_{i}=1$ and run the regression

$$
Y_{i, t}-\hat{\delta}_{i} Y_{i, t-1}=a_{i}+\sum_{j=1}^{p} \varphi_{i j} \Delta Y_{i, t-j}+\varepsilon_{i, t} .
$$

Construct the sample covariance matrix $\hat{\Omega}=(T-p-1)^{-1} \sum_{t=p+1}^{T} \hat{\varepsilon}_{t} \hat{\varepsilon}_{t}^{\prime}$, where $\hat{\varepsilon}_{t}=$ $\left(\hat{\varepsilon}_{1, t}, \ldots, \hat{\varepsilon}_{N, t}\right)^{\prime}$ are the vectors of residuals from the previous regression.

3. Project $\left(Y_{i, t}-c_{i, t}\right)$ and $\left(Y_{i, t-1}-c_{i, t-1}\right)$ on the lagged first differences

$$
\begin{aligned}
\left(Y_{i, t}-c_{i, t}\right) & =\sum_{j=1}^{p} \phi_{i j} \Delta Y_{i, t-j}+\xi_{i, t}, \\
\left(Y_{i, t-1}-c_{i, t-1}\right) & =\sum_{j=1}^{p} \zeta_{i j} \Delta Y_{i, t-j}+\xi_{i, t-1} .
\end{aligned}
$$

4. Define $\hat{\omega}_{i j}^{\prime}$ as the $i j^{t h}$ element of $\hat{\Omega}^{-1}$, one can now obtain the pooled FGLS estimator of $\delta$ and the associated t-statistic as

$$
\begin{aligned}
\hat{\delta}_{f g l s r m a} & =\frac{\sum_{i=1}^{N} \sum_{j=1}^{N} \hat{\omega}_{i j}^{\prime} \sum_{t=p+1}^{T} \hat{\xi}_{i, t-1} \hat{\xi}_{j, t}}{\sum_{i=1}^{N} \sum_{j=1}^{N} \hat{\omega}_{i j}^{\prime} \sum_{t=p+1}^{T} \hat{\xi}_{i, t-1}^{2}}, \\
t_{f g l s r m a} & =\frac{\hat{\delta}_{f g l s r m a}-1}{\sqrt{\sum_{i=1}^{N} \sum_{j=1}^{N} \hat{\omega}_{i j}^{\prime} \sum_{t=p+1}^{T} \hat{\xi}_{i, t-1}^{2}}}
\end{aligned}
$$

Sul (2006) shows that the $t_{f g l s r m a}$ converges to a Dickey-Fuller distribution, and he provides finite sample critical values to account for finite sample bias.

Similar to Breitung and Das (2006), Sul's (2006) $t_{\text {fglsrma }}$ effectively tests for a unit root in the data. To test for a unit root in the common component, Sul (2006) proposes to apply a recursive mean adjusted ADF test to the cross-sectional averages of the data, $\bar{Y}_{t}=$ $N^{-1} \sum_{i=1}^{N} Y_{i, t}$. Following Hansen (1995), he further suggests to augment this test using covariates to increase power. The steps of the procedure are similar to the ones outlined above, and the resulting t-statistic is denoted as $t_{c r m a}$. Sul (2006) provides some evidence that his test is precise and powerful, especially when $T$ is larger than $N$, a case for which it has been designed.

\subsection{Differences and similarities}

This section discusses differences and similarities of the panel unit root tests relying on a factor structure, presented in Sections 2.1, 2.2 and 2.3. As should have become clear, the Bai and Ng (2004a) approach is broader than that of Pesaran (2005) and Moon and Perron (2004), which are basically identical in terms of the assumed dynamic structure of the data, but differ in terms of tests performed and test statistics used. While Bai and Ng (2004a) allow the non-stationarity of the data to come from common or idiosyncratic sources, the 
Pesaran (2005) and Moon and Perron (2004) approaches assume common and idiosyncratic stochastic trends under the null hypothesis.

This point becomes obvious when we look at the restrictions the different DGPs place on the model given in (1). Pesaran (2005) implicitly restricts the dependence of the data on the common shocks, $D_{i}(L)$, and on the idiosyncratic shocks, $C_{i}(L)$ to $\Delta_{i}(L) \Phi(L)$, where $\Delta_{i}(L)=\sum_{j=0}^{\infty} \delta_{i}^{j} L^{j}$ and $\Phi(L)$ is the inverse of the lag polynomial of the composite error term. The restrictions implied by Moon and Perron (2004) require $D_{i m}(L)$ to be the $m^{\text {th }}$ element of $\Delta_{i}(L) \lambda_{i}^{\prime} \Phi(L)$ and $C_{i}(L)$ to be equal to $\Delta_{i}(L) \Gamma_{i}(L)$. Now, under the unit root null hypothesis, $\delta_{i}=1$ for all $i$ and so $\Delta_{i}(L)$ becomes the partial sum operator. The assumptions made on the lag polynomials $\Phi(L)$ and $\Gamma_{i}(L)$ then assure that all partial sums contain a unit root $^{10}$. Bai and Ng's (2004a) assumptions restrict (1) such that $D_{i m}(L)=\lambda_{i} \Phi_{m}(L) \sum_{j=0}^{\infty} L^{j}$, where $\Phi_{m}(L)$ is the $m^{t h}$ column of $\Phi(L)$, and $C_{i}(L)=\Delta_{i}(L) \Gamma_{i}(L)$. The assumptions on $\Gamma_{i}(L)$ ensure that the idiosyncratic part of the data will contain a unit root if $\delta_{i}=1$, while the assumptions on $\Phi(L)$ guaranty $k_{1}$ common stochastic trend. Hence, under the DGPs assumed by Pesaran (2005) and Moon and Perron (2004) the order of integration is the same for the idiosyncratic and the common component of the data, while Bai and Ng (2004a) allow them to differ.

Consequently, Pesaran's (2005) Moon and Perron's (2004) null hypothesis corresponds to a special case of Bai and Ng's (2004a) setup, namely where $k_{1} \geq 1$ and all idiosyncratic errors are I(1). Bai and Ng (2004a) do not explicitly define null and alternative hypotheses for their analysis. Their methodology enables one to separately test different properties of the data at hand. Due to consistent estimation of the (unobserved) common factors and idiosyncratic errors, it is possible to determine the source of observed non-stationarity. Also, instead of formulating a rather general alternative like some $Y_{i, t}$ are stationary (as do the other two approaches), the PANIC approach supplies the tools to assess which data series and which data components are $\mathrm{I}(0)$ and $\mathrm{I}(1)$.

As already mentioned, Pesaran (2005) and Moon and Perron (2004) assume the same order of integration for the common factor and idiosyncratic component. That is, the common factor and idiosyncratic processes are either both unit root processes or both stationary. One might expect that this assumption has a positive impact on the power of the tests. However, the tests are implemented in such a way that they in fact test for a unit root in the idiosyncratic components. Pesaran's (2005) test statistics account for the common factor by including the cross-sectional mean of $Y_{i, t}$ and its first difference, whereas Moon and Perron (2004) defactor the data prior to testing. This has been shown by Breitung and Das (2006) and forcefully argued by Bai and Ng (2006).

The CIPS test of Pesaran (2005), the tests of Moon and Perron (2004) and the $P_{\hat{E}}^{c, \tau}$ statistics of Bai and Ng (2004a) have the same null hypothesis, namely that the defactored data are unit root processes for all $i$. Also, all three approached use a heterogenous alternative,

\footnotetext{
${ }^{10}$ Although Moon and Perron (2004) allow $\operatorname{rank}(\Phi(1))<K$, they assume at least one integrated common factor under the null.
} 
namely that some series have a unit root and some do not. However, Moon and Perron (2004) use a pooled estimator of the first order autoregressive coefficient $\delta_{i}$ in the construction of their statistics. The individual specific $C A D F$ test of Pesaran (2005) and the $A D F_{\hat{E}}^{c, \tau}$ tests of Bai and Ng (2004a) for a unit root in the idiosyncratic component for a given $i$, and the alternative hypothesis is stationarity of that component.

All three type of tests are designed for large $N$ and $T$ due to the estimation of the common factor(s) either by using principle components or by including the cross-sectional mean as proposed by Pesaran (2005).

The three approaches also differ in the way they treat cointegration. While Pesaran (2005) and Moon and Perron (2004) exclude the possibility of cointegration among the $Y_{i, t}$, as well as between the observed data and the common factors, Bai and Ng (2004a) include both possibilities in their model. In particular, if $k_{1} \geq 1$ and $E_{i, t}$ is stationary for some $i$, then the observed data and the common factors are cointegrated for those $i$ with cointegrating vector $\left(1,-\lambda_{i}^{\prime}\right)^{\prime}$. Furthermore, if all idiosyncratic errors are $\mathrm{I}(0)$, then the orthogonalization matrix used by Moon and Perron (2004) to eliminate the common factors, $Q_{\Lambda}$, serves as cointegration matrix for the $Y_{i, t}$. So, Bai and Ng's (2004a) procedure can be used as a cointegration test ${ }^{11}$, using the null hypothesis $k_{1} \geq 1$ and all idiosyncratic errors are stationary ${ }^{12}$.

Deterministic components are also treated differently in the three papers. While Pesaran (2005) and Bai and Ng (2004a) suggest tests for models that include either a constant or a linear trend, Moon and Perron (2004) propose their test only in the presence of a restricted constant. Their analysis concludes that the test has no local power in the presence of unrestricted deterministic components. The local power properties of the Pesaran's (2005) and Bai and Ng's (2004a) test statistics have not been analyzed analytically so far.

The factor structure used by all three approaches is a convenient form to model crosscorrelation, or even cointegration between panel members. Therefore, the (necessary for pooled testing) assumption of independence between the elements of $F$ and $E$ is far less restrictive than the assumption of independent cross-sections, underlying the IPS and LLC test. In terms of computational burden, all procedures are rather easy to implement. Pesaran (2005) provides tables with critical values for his tests. The PANIC procedure of Bai and $\mathrm{Ng}(2004 \mathrm{a})$ also requires some tabulated critical values for the rank test statistics $M Q_{(\cdot)}^{c}$ and $M Q_{(\cdot)}^{f}$, as well as for the $A D F_{\hat{E}_{i, t}}^{\tau}$ statistic. Also, a procedure to calculate the p-values of $A D F_{\hat{E}_{i, t}}^{c}$ and $A D F_{\hat{E}_{i, t}}^{\tau}$ is needed to implement the suggested pooled tests.

An important aspect in application is the selection of the number of common factors $K$, in particular for the tests presented in Sections 2.2 and 2.3, as they rely on consistent estimation of the factor structure used to model cross-sectional correlation. For that purpose, the number of common factors $K$ has to be estimated. This is discussed in Bai and $\mathrm{Ng}$ (2002)

\footnotetext{
${ }^{11}$ What is meant here is a cointegration test between panel members, in contrast to panel cointegration tests. The latter ones are used to test for cointegration between several variables for the same $i$.

${ }^{12}$ Note that the null hypothesis for the ADF tests using the estimated error terms remains that of nonstationarity. Rejecting the unit root hypothesis for all $i$ is thus one part of not rejecting cointegration between panel members.
} 
for a factor model as given by (13) with stationary errors, and also briefly treated in Moon and Perron (2004).

The statistics introduced in Section 2.4 provide an alternative to panel unit root testing for DGPs with cross-sectional dependence, which assume a common factor structure but do not exploit it to its full extend. Breitung and Das's (2006) statistics test the homogenous unit root null hypothesis against the homogenous stationary alternative, while Sul (2006) considers the same null but allows for heterogeneity under the alternative. For the DGPs in (19) and (20), the GLS statistics which effectively test for a unit root in the data have the drawback that they are only feasible when $N<T$.

\section{Small sample performance: Monte Carlo results}

\subsection{Monte Carlo simulation setup}

In this section we study the small sample performance of the tests proposed by Pesaran (2005), Moon and Perron (2004) and Bai and Ng (2004a) for various types of DGPs. Furthermore, we consider the robust OLS t-test $t_{r o b}$ and the GLS t-test $t_{G L S}$ described in Breitung and Das (2006) and the recursive mean adjusted FGLS test $t_{\text {prmagls }}$ and the recursive mean adjusted test for the average data proposed by Sul (2006). All considered DGPs are special cases of (1) and with one exception have the following structure which corresponds to Bai and Ng's (2004a) framework:

$$
\begin{aligned}
Y_{i, t} & =\lambda_{i}^{\prime} F_{t}+E_{i, t}, \\
F_{m, t} & =\varphi F_{m, t-1}+f_{m, t}, \\
E_{i, t} & =\delta_{i} E_{i, t-1}+e_{i, t},
\end{aligned}
$$

with $i=1, \ldots, N, t=1, \ldots, T$ and $m=1, \ldots, K$. We consider three different values for $N$ and $T$ each, namely 20,50 and 100. The method of principle components estimates the space spanned by the common factors when $N$ is large. We have chosen $N$ and $T$ at least equal to 20 to assure that common factors are estimated with sufficient precision or approximated reasonably well by cross-sectional averages. Notice that the regularity condition $N \neq T$ needed for some tests is not satisfied in some cases. First a single common factor is considered, which is generated by a first order autoregression, or a random walk when $\varphi=1$. We also consider the case of two common factors which are generated using the same parameter values for $\varphi$ and $\sigma_{f}^{2}$, but different drawings for the error terms. The idiosyncratic terms $E_{i, t}$ are also generated by a first order autoregression or random walk with first order moving average, depending on whether or not $\delta_{i}=1$.

In addition, a DGP as assumed by Pesaran (2005) and Moon and Perron (2004) is used:

$$
\begin{aligned}
& Y_{i, t}=\delta_{i} Y_{i, t-1}+u_{i, t}, \\
& u_{i, t}=\lambda_{i} f_{t}+e_{i, t} .
\end{aligned}
$$


In (25) and (26) the error terms are generated as MA(1) processes such that

$$
\begin{aligned}
f_{m, t} & =\eta_{m, t}+\gamma_{m} \eta_{m, t-1}, \\
e_{i, t} & =\varepsilon_{i, t}+\rho_{i} \varepsilon_{i, t-1} .
\end{aligned}
$$

The shocks are drawn from independent normal distributions, such that $\eta_{t} \sim$ i.i.d.N $\left(0, \Sigma_{f}^{2}\right)$, with $\Sigma_{f}^{2}=\sigma_{f}^{2} I_{K}$, and $\varepsilon_{i, t} \sim$ i.i.d.N $(0,1)$. We consider three different values for the signal-tonoise ratio, such that $\sigma_{f}^{2}=0.5,1$ and 2. The MA parameters $\gamma_{m}$ and $\rho_{i}$ are independently, uniformly distributed on $[0.2,0.5]$. The factor loading $\lambda_{i}$ are uniformly distributed on $[-1,3]^{13}$.

Three different types of non-stationarity are considered as null hypothesis, as well as different settings for the stationary alternative hypothesis. In particular, we consider the following 5 cases, where 1 to 4 use the DGP given by (25) and 5 uses DGP $(26)^{14}$ :

1. Common and idiosyncratic unit roots

$$
H_{0}^{A}: \varphi=1, \text { and } \delta_{i}=1 \text { for all } i
$$

2. Common unit root, nearly stationary idiosyncratic components

$$
H_{0}^{B}: \varphi=1, \text { and } \delta_{i} \sim U[0.8,1] \text { for all } i
$$

3. Stationary common component, integrated idiosyncratic components

$$
H_{0}^{C}: \varphi=0.95, \text { and } \delta_{i}=1 \text { for all } i
$$

4. Stationary common and idiosyncratic components

$$
H_{A}^{A}: \varphi=0.95 \text { and } \delta_{i} \sim U[0.8,1] .
$$

5. Stationary data using a DGP as given by (26) with heterogenous roots

$$
H_{A}^{E}: \delta_{i} \sim U[0.8,1] \text { for all } i
$$

The reported rejection frequencies are based on 5\% nominal size. For Pesaran's (2005) $C A D F$ and $C I P S$ we use the critical values reported in Tables $1 \mathrm{~b}$ and $3 \mathrm{~b}$ of his paper. Results for Moon and Perron's (2004) statistics and Bai and Ng's (2004a) $P_{\hat{E}}^{c}$ statistic are based on a critical value from the standard normal distribution. Rejection frequencies of the $A D F_{\hat{E}}^{c}$ and $A D F_{\hat{F}}^{c}$ statistics are obtained using the critical values from DF distributions for

\footnotetext{
${ }^{13}$ Consistency of the test procedure of Pesaran (2005) requires a non-zero mean for the factor loadings. This assumption is not necessary for the other approaches.

${ }^{14}$ Please note that under setup $1(25)$ and (26) are equivalent. In cases 2,4 and 5 we have stationarity provided $\delta_{i} \neq 0$.
} 
the no constant and constant only cases, respectively. Critical values for the $M Q_{c}^{c}$ and $M Q_{f}^{c}$ are provided in Table 1 of Bai and Ng (2004a).

Similar to Moon and Perron (2004), we use the Andrews-Monahan (1992) estimator employing the quadratic spectral kernel in the estimation of the nuisance parameters for the $t_{a}^{*}$ and $t_{b}^{*}$ statistics. For Bai and Ng's (2004a) $A D F_{\hat{E}}^{c}$ and $A D F_{\hat{F}}^{c}$ and Pesaran's (2005) $C A D F$ and $C I P S$ we use the Akaike information criterion (AIC) to determine the lag length, starting with a maximum lag length of $p_{\max }=6$. For the test of Sul (2006) and Breitung and Das (2006) we use the Bayesian information criterion (BIC). For the $M Q_{c}^{c}$ statistic we use the Bartlett kernel with a bandwidth as suggested in Andrews (1991). The lag length for the $M Q_{f}^{c}$ statistic is determined using the criteria proposed by Aznar and Salvador (2002).

\subsection{Monte Carlo results}

A general finding is that the presence of serial correlation ${ }^{15}$ leads to size distortions for almost all statistics when $T$ is small, which can be quite strong in some cases and even persist for $T=100$. For a single common factor, the signal-to-noise ratio seems to have little to no effect on the tests proposed by Pesaran (2005) and Bai and $\mathrm{Ng}$ (2004a). For 2 common factors in the DGP, Bai and Ng's (2004a) $M Q_{c}^{c}$ and $M Q_{f}^{c}$ statistics usually select maximum possible number of common stochastic trends, leading to low size and low power for these tests when the auto-regressive root is close to unity. The GLS statistics of Breitung and Das (2006) and Sul (2006) behave quite similarly. Sul's (2006) $t_{c r m a}$ statistic applied to the cross-sectional averages of the data shows a bad performance, with huge size distortions but small power for all combinations of $N$ and $T$. Furthermore, of the considered test procedures only the approach suggested by Bai and Ng (2004a) can detect a unit root resulting only from non-stationary common factors.

The results in Table 1 are obtained for the case where a unit root is present in the common factor and in all idiosyncratic errors. Both statistics proposed by Pesaran (2005), the $C A D F^{16}$ and the $C I P S$ test show size distortions when $T$ is small (20), which are stronger for the CIPS test. As $T$ increases those size distortions are reduced, and for $T=100$ the tests are only slightly over-sized for some combinations of $N$ and $T$. Both statistics proposed by Moon and Perron (2004) show slight size distortions which seem to increase with the signal-to-noise ration. The size distortions are decreasing in $T$ and higher for $t_{b}^{*}$ than for $t_{a}^{*}$. Bai and Ng's $A D F_{\hat{E}}^{c}$ and $A D F_{\hat{F}}^{c}$ statistics for the extracted individual idiosyncratic error series and the single common factor respectively, are oversized for small $T(=20)$ but size distortions decrease as $\mathrm{T}$ gets large. The pooled statistic $P_{\hat{E}}^{c}$ has strong size distortions when $T$ is small and size seems to increase in $N$. For $T=100$, size rages from 0.12 to 0.18 for the different values of $N$. Breitung and Das's (2006) $t_{r o b}$ is under-sized for small $T$ with rejection frequencies increasing in $T$ but decreasing with $N$, leading to rejection frequencies between

\footnotetext{
${ }^{15}$ Results for the case of i.i.d. $N(0,1)$ error terms $e_{i, t}$ and $f_{T}$ in $(25)$ are not included in this version of the paper. They are are available at http://www.personeel.unimaas.nl/J.Urbain/.

${ }^{16}$ Entries for the CADF-statistics are average rejection frequencies of the individual unit root tests.
} 
0.00 and 0.10. The $t_{g l s}$ has a size of about 0.05 for $N=20$ and is under-sized for $N=50$. Sul's $T_{\rho f g l s r m a}$ tests behaves similarly, but rejection frequencies are slightly higher with a size above 0.05 for $T=50$. The $t_{\text {crma }}$ test for the cross-sectional averages has a size between 0.50 and 0.55 .

\section{INSERT TABLES 1-2 ABOUT HERE}

Table 2 considers the case of a unit root in the common factor and near-unit roots in the idiosyncratic factors, i.e. the case of cross-member cointegration. Pesaran's (2005) CADF statistic has an average rejection frequency of about 0.32 for $T=20$ and between 0.17 and 0.21 for larger $T$. The rejection frequencies of the CIPS test are high and go to 1 for large $N$ and $T$. Both statistics proposed by Moon and Perron (2004) have rejection frequencies of 1 for $T>50$. Bai and Ng's $A D F_{\hat{E}}^{c}$ statistic has an average power increasing from about 0.23 to 0.48 as both $N$ and $T$ increase. The pooled $P_{\hat{E}}^{c}$ test has a power of 1 for almost all combinations of $N$ and $T$ considered. The $A D F_{\hat{F}}^{c}$ tests has some size distortions, but rejection frequencies decrease from about 0.40 for $T=20$ to 0.07 to 0.10 for $T=100$. Rejection frequencies for Breitung and Das's (2006) $t_{r o b}$ statistics decrease for higher signal-to-noise ratios, whereas they increase with $T$. The $t_{g l s}$ statistic has relatively high rejection frequencies (close to or above 0.90 for $N=50, T=100)$, increasing with $N$ and $T$. Sul's (2006) $t_{\rho f g l r m a}$ statistic behaves similarly but has slightly lower rejection frequencies. Rejection frequencies for $t_{\text {crma }}$ are between 0.56 and 0.66 .

\section{INSERT TABLE 3 ABOUT HERE}

Table 3 covers the case of integrated idiosyncratic errors combined with a stationary common factor. The statistics proposed by Pesaran (2005) and Moon and Perron (2004) behave similar to the case of I(1) idiosyncratic and common component (Table 1), but size is slightly reduced for the CIPS test, which is now under-sized for $T=100$, while $t_{a}^{*}$ and $T^{*} b$ have slightly higher rejection frequencies. Also, Bai and Ng's (2004a) $A D F_{\hat{E}}^{c}$ and $P_{\hat{E}}^{c}$ tests have sizes close to the one shown in Table 1. The power of the $A D F_{\hat{F}}^{c}$ is smaller than 0.20 for $T \geq 50$. Breitung and Das's (2006) $t_{r o b}$ test has size increasing in $T$ but decreasing in $N$ and the signal-to-noise ratio. The $t_{g l s}$ and Sul's (2006) $t_{\text {fglsrma }}$ tests are slightly over-sized for $N=20$ and under-sized for $N=50$, where the size for the later is slightly higher. The $t_{c r m a}$ test again has rejection frequencies of about 0.50 .

\section{INSERT TABLES 4-5 ABOUT HERE}

Tables 4 and 5 consider stationary data. For Table 4 the DGP is given by (25) with I(0) idiosyncratic and common components. Pesaran's (2005) $C A D F$ has low power while the power of the CIPS test is relatively high and increasing in $N$, reaching 1 for $N, T=100$. Moon and Perron's (2004) tests both have power of 1 for most considered combinations of $N$ and $T$. The average power of Bai and Ng's (2004a) $A D F_{\hat{E}}^{c}$ is relatively low (0.52 for 
$N, T=100)$ while the pooled test $P_{\hat{E}}^{c}$ has a power of 1 for $N>20$ or $T>20$. The power of the $A D F_{\hat{F}}^{c}$ is low. Breitung and Das's (2006) $t_{r o b}$ has power increasing in $T$ but decreasing in $N$ and the signal-to-noise ratio. The two considered GLS statistics, $t_{g l s}$ and Sul's (2006) $t_{\text {fglsrma }}$ have a high power, which is (close to) 1 for $T=100$. Rejection frequencies for the $t_{\text {crma }}$ tests are between 0.56 and 0.67 .

Table 5 considers stationary data generated using (26). The results are similar to Table 4 for mosts tests. However, the power for Moon and Perron's (2004) $t_{a}^{*}$ and $t_{b}^{*}$ is slightly reduced for higher signal-to-noise ratios. Bai and Ng's (2004a) $A D F_{\hat{F}}^{c}$ has a higher power now, but it is still relatively low. Rejection frequencies for Sul's $t_{c r m a}$ test are reduced as well, in particular for $N=50$ where power is even below the nominal $5 \%$ size for a signal-to-noise ratio of 2 .

Tables 5 to 10 present the results of the simulations with 2 common factors. We no longer report the results for Bai and Ng's (2004a) $A D F_{\hat{E}}^{c}$ statistic as they are basically identical to those obtained for the single common factor case. For the two rank statistics $M Q_{c}^{c}$ and $M Q_{f}^{c}$ we report the proportion of replications for cases where the true number of common stochastic trends were selected, i.e. for which $\hat{k}_{1}=2$ if $\varphi=1$ or $\hat{k}_{1}=0$ otherwise. For both statistics we use the simulated $5 \%$ critical values reported by Bai and $\mathrm{Ng}$ (2004a). The number of common factors is assumed to be known and is used in the derivation of the Moon and Perron (2004) and Bai and Ng (2004a) statistics. Pesaran (2005) only considers the case $K=1$ for his procedure, so the results reported for the CADF and CIPS statistics indicate their robustness to this assumption. Although Breitung and Das's (2006) consider the possibility of accounting for the factor structure when estimating the covariance matrix for the GLS statistic, we employ the more general version where the factor structure is not imposed.

Table 6 presents the findings for the combination of two common stochastic tends with non-stationary idiosyncratic errors. Pesaran's (2005) CADF statistic has slightly higher size than in the single factor case, but the pooled CIPS statistic experiences size distortion that increase in $N$ as well as in the signal-to-noise ratio. The $t_{a}^{*}$ and $t_{b}^{*}$ statistics of Moon and Perron (2004) behave similar to $K=1$ but size distortions are slightly decreased. Bai and Ng's (2004a) $P_{\hat{E}}^{c}$ statistic now has smaller size distortions for $T=20$ than in the single common factor case. Their two rank statistics $M Q_{c}^{c}$ and $M Q_{f}^{c}$ show good properties with a frequency close to 1 of selecting the correct number of common stochastic trends. Results for the tests of Breitung and Das (2006) and Sul (2006) are similar to those derived for a single common factor.

The combination of non-stationary common factors with stationary idiosyncratic errors is considered in Table 7. In this setup there is again cross-member cointegration in the panel, but with two common trends driving the non-stationarity. As in the single factor case the Moon and Perron (2004) statistics have high rejection frequencies which are mostly close to 1 for $T \geq 50$. The $P_{\hat{E}}^{c}$ statistic now has still high power but it is slightly reduced for small $N, T$, when compared to the single factor case. Also, the two rank statistics select the correct 
number of I(1) factors for all considered sample sizes. The CADF and CIPS tests have reduced rejection frequencies when compared to the single common factor case (Table 2). Rejection rates for the CIPS test are also decreasing as the signal-to-noise ratio increases. It seems that de-factoring the data by augmenting the ADF-regression with cross-sectional averages no longer removes the common trends completely. Again, the results for the tests of Breitung and Das (2006) and Sul (2006) are similar to those obtained for $K=1$.

\section{INSERT TABLES 6-7 ABOUT HERE}

Table 8 considers the case of non-stationary idiosyncratic errors combined with stationary common factors. The statistics of Pesaran (2005) and Moon and Perron (2004) based on de-factored data now have slightly higher sizes than in the single factor case. Also, rejection frequencies for the $t_{a}^{*}, t_{b}^{*}$ and $C I P S$ tests now increase with the signal-to-noise ratio. The $P_{\hat{E}}^{c}$ statistic of Bai and Ng (2004a) has a slightly reduced rejection frequency but is still over-sized. The rank statistics $M Q_{c}^{c}$ and $M Q_{f}^{c}$ however fail to reject common stochastic trends. Sizes for the tests proposed by Breitung and Das (2006) and for Sul's (2006) $t_{f g l s r m a}$ are slighly increased when compared to the single common factor case.

\section{INSERT TABLES 8 ABOUT HERE}

Power results for the stationary DGPs we consider are presented in Tables 9 and 10. Table 9 give the results when the DGP given in (25) is employed. For Pesaran's CADF and CIPS statistics power is reduced when compared to the one common factor case, and furthermore the power for the CIPS test decreases as the signal-to-noise ratio increases. The $t_{a}^{*}, t_{b}^{*}$ and

$P_{\hat{E}}^{c}$ tests have high power which is 1 for $T>20$, while the rank tests $M Q_{c}^{c}$ and $M Q_{f}^{c}$ fail to reject non-stationarity for the extracted common factors. The power decrease of the $t_{r o b}$ test as the signal-to-noise ratio increases is stronger than in the single factor case, while the 2 GLS statistics have good power. When the DGP given in (26) is used (Table 10), the power of the CIPS and $t_{r o b}$ tests is increased, while the two statistics proposed by Moon and Perron (2004) and the two GLS tests have slightly reduced power. Also, the rejection frequencies for Sul's $t_{c r m a}$ is reduced, even below the nominal $5 \%$ level in some cases.

\section{InsERT TABLES 9-10 ABOUt HeRE}

Tables 11-15 present results for the tests of Moon and Perron (2004) and Bai and Ng (2004a) where the DGP contains 2 common factors, but we have misspecified the number of common factors in the estimation by setting it either equal to 1 (l.h.s. panel) or to 3 (r.h.s. panel). As for the one factor case, the $A D F_{\hat{F}}^{c}$ for the extracted common factor has some size distortions and low power. The rank tests $M Q_{c}^{c}$ and $M Q_{f}^{c}$ fail to detect the true number of common stochastic trends and usually select the maximum possible number $\left(\hat{k}_{1}=3\right)$. For the $P_{\hat{E}}^{c}$ size distortions are lower and power is higher when too many factors are extracted than when too few factors are accounted for. Similarly, for Moon and Perron's (2004) test 
power is better when the number of common factors is overestimated. Both tests experience increased size distortions, but these are better for small $N$ when only a single common factor is included in the estimation and better for large $N$ when 3 factors are incorrectly accounted for.

\section{INSERT TABLES 11-15 ABOUT HERE}

From the Monte Carlo simulations, several general conclusions can be drawn. The presence of serial correlation in the error term leads to size distortions which can be quite large in small samples. The Moon and Perron (2004) tests appear to be somewhat more powerful than the Pesaran's (2005) CIPS test. In general, both types of tests have similar small sample behavior. The $t_{a}^{*}$ test of Moon and Perron (2004) has less size distortions than the $t_{b}^{*}$ test.

To test for the presence of a unit root in the idiosyncratic components, the $P_{\hat{E}}^{c}$ test of Bai and $\mathrm{Ng}$ (2004a) should be preferred to their $A D F_{\hat{E}}^{c}$ test. If the unit root in the data in fact is due to a unit root in the common factors, only the Bai and Ng (2004a) tests have decent properties. The tests put forward by Pesaran (2005) and Moon and Perron (2004) are designed to test for the presence of unit roots in the de-factored data. In the case of cross-sectional dependencies due to a single common factor, the two types of procedures have similar small sample properties. Whereas Pesaran's (2005) tests account for the effect of this common factor by including cross-sectional averages (of first differences and lagged levels) in the regression yielding the $C A D F$ statistic, Moon and Perron (2004) compute their test statistics using de-factored data. While the Moon-Perron approach applies to the multifactor case, augmenting regressions with cross-sectional averages will not fully account for the presence of several factors. Therefore, in the presence of one common factor Pesaran's (2005) tests might be preferred, given that they can be computed in a straightforward way and that they have similar small sample properties to the Moon and Perron (2004) procedures. In multi-factor settings the latter tests are to be preferred. In that case, including cross-sectional means will not lead to the elimination of all cross-sectional dependency, so that a central limit theorem might not apply. As shown, the $C A D F$ and $C I P S$ tests suffer from size distortions and reduced power. Another difference between Pesaran (2005) and Moon and Perron (2004) is that Pesaran's (2005) CIPS test pools the statistics whereas Moon and Perron (2004) use a pooled estimator to base their statistics on.

The Bai and Ng (2004a) tests have been proposed for a different purpose. They provide tools to find out whether there are unit roots in the common factors and/or in the idiosyncratic components. However, their tests for common stochastic trends, i.e. $A D F_{\hat{F}}^{c}, M Q_{c}^{c}$ and $M Q_{f}^{c}$ have low power.

The GLS statistics by Breitung and Das (2006) and by Sul (2006) which do not rely on modelling the cross-sectional correlation using a factor models have good properties but fail to detect a unit root introduced only through the common factors in the data.

We have not studied the issue of which test to choose if the common factor model representation is not appropriate to describe cross-sectional dependence. Bootstrap unit root tests 
might be used in such an instance, but this question is left for future research.

\section{An illustrative application: Testing for PPP using the new approaches}

This section presents an application of the new panel unit root tests described in Section 2 to illustrate their use in an empirical study of the validity of purchasing power parity (PPP). For this purpose we consider the potential existence of a unit root in real exchange rate series that are constructed as

$$
Y_{i, t}=s_{i, t}-p_{t}^{*}+p_{i, t},
$$

where $s_{i, t}$ is the ln of country $i$ 's nominal exchange rate versus some numeraire currency, $p_{t}^{*}$ is the ln of the aggregate price level in the numeraire country, and $p_{i, t}$ is the ln of country $i$ 's domestic aggregate price level.

The numerous analyzes of PPP in the literature do not come to a common conclusion with respect to PPP. Some studies report stronger rejection of the unit root null, if the German Mark instead of the US Dollar is used as a numeraire currency. Also, studies using univariate unit root or cointegration tests reject it, while tests using panel methods as the LLC or IPS test tend to find evidence in favor of PPP, see for example Oh (1996). However, as was already discussed in the introduction, several studies have analyzed the properties of early panel unit root tests in the presence of cross-sectional dependence since then, and argued against their use for PPP tests. Lyhagen (2000) analytically derives the cross-correlation structure in a panel of real exchange rates, constructed with a common numeraire country. He also derives the effect of the common stochastic trend in the data introduced by the numeraire on the limiting distributions of various panel statistics. In Monte Carlo simulations, he finds size distortions similar to those reported by Banerjee et al. ((2004) and (2005)).

In the analysis presented in this section, monthly data from 14 European countries is considered. The data set includes information on the nominal exchange rates of local currency versus US Dollar (\$US) for Austria, Denmark, Finland, France, Germany, Greece, Italy, the Netherlands, Norway, Portugal, Spain, Sweden, Switzerland and the UK. Furthermore, the Consumer Price Index (CPI) as a proxy for aggregate price levels is included for those 14 countries and the US. The sample includes monthly observations on all variables for the period from February 1986 to September 2002, so 200 observations. For such a sample, one can expect to find high correlation between panel units, due to a high degree of economic integration and political co-operation. As far as monetary policy is concerned, the most important mechanism of co-operation is the European Monetary System (EMS), to which some panel members belong, and which finally led to the introduction of the Euro as a common currency in some countries.

As a starting point of the analysis, the real exchange rate series are individually tested for a unit root using an ADF test. The lag length $p$ is set to 12 for all countries. The individual 
ADF statistics are shown in Table 16. Only for the UK, the unit root null can be rejected for both real exchange rate series. Using the real exchange rate versus DM, also for Switzerland the ADF test rejects at a 5\% significance level. These findings are representative for those of studies using univariate tests. The problem here is that it remains unclear whether the non-rejection of the unit root is due to a failure of PPP, or the low power of the ADF test against near unit root alternative.

Next, the panel unit root tests described in Section 2 are performed. For each test, it is assumed that a single common factor is present in the data. Given that the real exchange rate series are constructed using a common base currency, this assumption seems reasonable. For the tests of Pesaran (2005) and Moon and Perron (2004), the data representation in (2)-(3) is assumed to be valid. The results of the $C A D F_{i}$ tests suggested by Pesaran (2005) are given in Table 16, and Table 17 presents Pesaran's (2004) CIPS statistic and those proposed by Moon and Perron (2004). Except for the French real exchange rate when measured against the German Mark, the $C A D F_{i}$ statistics fail to reject the unit root null. Also, the pooled CIPS test does not reject the null in both panels. This provides some evidence against PPP. The $t_{a}^{*}$ and $t_{b}^{*}$ statistics of Moon and Perron (2004) do not provide such a clear picture. While the former one rejects PPP in both panels, the latter one does not reject it.

The results for the panel unit root tests proposed by Breitung and Das (2006) and by Sul (2006) are given in Table 17. While both tests by Breitung and Das (2006) do not reject the unit root null for both panels of real exchange rates, the $t_{\text {fglsrma }}$ test of Sul (2006) does reject the unit root when real exchange rates are constructed with Germany as base country.

\section{INSERT TABLES 16-17 ABOUT HERE}

For the application of the Bai and Ng (2004a) procedure, it is assumed that the data can be represented as in (13). With this representation, there is an interpretive problem. Clearly, if both $F_{t}$ and $E_{i, t}$ are stationary, the real exchange rate is stationary and PPP holds in the long run at least. Also, if both common and idiosyncratic components are I(1), PPP can be rejected. But, if just the common factors are non-stationary the real exchange rate series are pairwise cointegrated along the cross-section but individually non-stationary, so that PPP in the usual sense does not hold between panel members and the base country. However, in the special case $\lambda_{i}=\lambda_{j}$, the cointegrating vector for $Y_{i, t}^{B}$ and $Y_{j, t}^{B}$ is $[1,-1]$, where the superscript $B$ denotes the base country. Then PPP holds between countries $i$ and $j$, since

$$
Y_{i, t}^{B}-Y_{j, t}^{B}=s_{i, t}^{B}-s_{j, t}^{B}+p_{i, t}-p_{j, t}=s_{i, t}^{j}+p_{i, t}-p_{j, t}=Y_{i, t}^{j} \sim I(0) .
$$

The results for the test statistics suggested by Bai and Ng (2004a) are presented in Table 17. Most of the individual test for the idiosyncratic errors, as well as the test for the common factor reject the unit root. Also, the pooled error test rejects the unit root for both panels of real exchange rates. This provides some evidence in favor of PPP. 


\section{Conclusion}

In this paper several panel unit root tests that account for cross section dependence assuming or using a common factor structure have been proposed in the literature, notably Pesaran (2004), Moon and Perron (2004) and Bai and Ng (2004a). There are often valid theoretical and empirical reasons why a common factor structure can be expected to yield sensible results. Therefore, panels with dynamic factors are of interest in economic modelling.

We have studied the three approaches to unit root testing in panels with dynamic factors, compared them in terms of DGP, tests, null and alternative hypotheses. We have studied the small sample behavior of the tests proposed in a common framework. We have discussed their use in econometric modelling and compared them with the tests proposed by Breitung and Das (2006) and Sul (2006). In addition, we have applied them in an empirical study of purchasing power parity.

The main conclusions are:

- In the case where the observed non-stationarity is only due to a non-stationary common factor, the individual series are pairwise cointegrated along the cross sectional dimension. Only the Bai and Ng (2004a) tests allow for this type of structure to be detected. In this case, the other considered tests tend to systematically reject the unit root that actually is present in the series.

- Pesaran's (2005) CADF and CIPS tests are indeed designed for testing for unit roots in the idiosyncratic components when cross-sectional dependence is due to a single common factor. The pooled CIPS test has better power properties than the individual specific CADF tests.

- The Moon and Perron (2004) tests which use de-factored data can account for multiple common factors. Therefore, their use has to be recommended when cross-section dependence is expected to be due to several common factors. The two tests proposed by Moon and Perron (2004) are found to have similar small sample power, but the $t_{a}^{*}$ statistic is found to have slightly smaller size distortions than the $t_{b}^{*}$. They were found to be more powerful than the Pesaran (2005) tests, which have the advantage to be easy to compute.

- The Bai and Ng (2004a) tests are designed to separately test for the presence of unit roots in the common factors and in the idiosyncratic components.

- From the Monte Carlo analysis, we conclude that the $P_{\hat{E}}^{c}$ tests is more powerful than the $A D F_{\hat{E}}^{c}$ in detecting unit roots in the idiosyncratic components, although the former can have strong size distortion when the time dimension of the panel is small.

- The $A D F_{\hat{F}}^{c}$ for testing for the presence of unit roots in a single common factor is found to have low power. Similarly, in a multi-factor setting, the $M Q_{c}^{c}$ and $M Q_{f}^{c}$ tests fail to distinguish high but stationary serial correlation from non-stationarity in the common 
factors. For the one factor model, Bai and Ng's (2004) $A D F c_{\hat{F}}$ test has better size than Sul's (2006) $t_{c r m a}$ test except when $N$ is small and $T$ is large, although the rejection frequencies are much higher than the nominal size.

- When the number of common factors is unknown and has to be selected, it is less harmful to include too many factors than too few in the test procedures of Bai and $\mathrm{Ng}$ (2004a) and Moon and Perron (2004).

- The considered GLS tests by Breitung and Das (2006) and Sul (2006) can be computed, i.e. when $N<T$. They provide good alternatives to test for unit roots in the data when the nonstationarity does not only come from the common factors. In the presence of cross-member cointegration the tests diverge.

As the tests by Pesaran (2005), Moon and Perron (2004) and the $A D F_{\hat{E}}^{c}$ and $P_{\hat{E}}^{c}$ by Bai and $\mathrm{Ng}$ (2004a) test for the presence of a unit root in the idiosyncratic components, it is worthwhile to compare their small sample properties.

- In the one-factor model, under the null hypothesis of a unit root in the idiosyncratic component, all tests perform well or reasonably well. The $P_{\hat{E}}^{c}$ test of Bai and Ng (2004a) over-rejects the null when the common factor is nonstationary.

- In the two-factor model with $\hat{K}=K=2$, surprisingly the $C A D F$ test of of Pesaran (2005) has a reasonable size. The $P_{\hat{E}}^{c}$ test by Bai and $\mathrm{Ng}(2004 \mathrm{a})$ has a reasonable size as well when the common factors have a near-unit root.

- In the two-factor model with $\hat{K}=1$ and with nonstationary common factors or with $\hat{K}=3$ and near-unit root common factor processes, the $A D F_{\hat{E}}^{c}$ test has a size close to the nominal one. The other tests are oversized. In other cases, all tests appear to be oversized.

- For all models considered, the $P_{\hat{E}}^{c}$ test by Bai and $\mathrm{Ng}$ (2004a) has power one or close to one. The power of the tests by Moon and Perron (2004) is in general somewhat lower than that of the $P_{\hat{E}}^{c}$ test. The power of the tests by Pesaran (2005) and of the $A D F_{\hat{E}}^{c}$ test of Bai and Ng (2004a) is much lower in most instances, however Pesaran's (2005) $C A D F$ test and Bai and Ng's (2004a) $A D F_{\hat{E}}^{c}$ test are individual specific statistics. In terms of finite sample power, the $P_{\hat{E}}^{c}$ test of Bai and $\mathrm{Ng}(2004 \mathrm{a})$ and the tests of Moon and Perron (2004) seem to be the preferred choice on the basis of this Monte Carlo study.

\section{References}

[1] Andrews, D.W.K. (1991), Heteroskedasticity and Autocorrelation Consistent Covariance Matrix Estimation, Econometrica 59, 817-858. 
[2] Andrews, D.W.K. and Monahan, C. (1992), An Improved Heteroskedasticity and Autocorrelation Consistent Covariance Matrix Estimator, Econometrica 60, 953-966.

[3] Aznar, A. and Salvador, M. (2002), Selecting the Rank of the Cointegration Space and the Form of the Intercept Using an Information Criterion, Econometric Theory 18, 926947.

[4] Bai, J. and Ng, S. (2002), Determining the number of Factors in Approximate Factor Models, Econometrica 70, 191-221.

[5] Bai, J. and Ng, S. (2004a), A PANIC Attack on Unit Roots and Cointegration, Econometrica $72,1127-1177$.

[6] Bai, J. and Ng, S. (2004b), A New Look at Panel Testing of Stationarity and the PPP Hypothesis, in Indentification and Inference in Econometric Models: Essays in Honor of Thomas J. Rothenberg, Don Andrews and James Stock (eds.), Cambridge University Press.

[7] Bai, J. and Ng, S. (2006), Panel Unit Root Tests with Cross-Section Dependence: A Further Investigation, Working Paper, Department of Economics, New York University.

[8] Banerjee, A., Marcellino, M. and Osbat, C. (2004), Some Cautions on the Use of Panel Methods for Integrated Series of Macro-Economic Data, Econometrics Journal 7, 322340.

[9] Banerjee, A., Marcellino, M. and Osbat, C. (2005), Testing for PPP: Should We Use Panel Methods?, Empirical Economics 30, 77-91.

[10] Breitung, J. and Das, S. (2006), Testing for Unit Roots in Panels with a Factor Structure, Econometric Theory, forthcoming.

[11] Chang, Y., 2002, Nonlinear IV Unit Root Tests in Panels with Cross-Sectional Dependency, Journal of Econometrics 110, 261-292.

[12] Choi, I. (2001), Unit Root Tests for Panel Data, Journal of International Money and Finance 20, 249-272.

[13] Flôres, R., Jorion, P., Preumont, P.-Y., Szafarz, A. (1999) Multivariate unit root tests of the PPP hypothesis, Journal of Empirical Finance 6, 335-353.

[14] Im, K.S., Pesaran, M.H., Shin, Y. (2003), Testing for unit roots in heterogenous panels, Journal of Econometrics 115, 53-74.

[15] Levin, A., Lin, C.F., Chu C.S.J. (2002), Unit root tests in panel data: asymptotic and finite-sample properties, Journal of Econometrics 108, 1-24.

[16] Lyhagen, J. (2000), Why not use standard panel unit root test for testing PPP, mimeo, Stockholm School of Economics. 
[17] Maddala, G.S. and Wu, S. (1999), A Comparative Study of Unit Root Tests with Panel Data and Cointegration Tests, Oxford Bulletin of Economics and Statistics 61, 631-652.

[18] Moon, H.R. and Perron, B. (2004), Testing for a Unit Root in Panels with Dynamic Factors, Journal of Econometrics 122, 81-126.

[19] O'Connell, P.G.J. (1998), The overvaluation of purchasing power parity, Journal of International Economics 44, 1-19.

[20] Oh, K.-Y. (1996), Purchasing Power Parity and unit root tests using panel data, Journal of International Money and Finance 15, 405-418.

[21] Pesaran, M.H. (2005), A Simple Panel Unit Root Test in the Presence of Cross Section Dependence, Cambridge University DAE Working Paper, number 0346.

[22] Phillips, P. and Sul, D. (2003), Dynamic Panel Estimation and Homogeneity Testing Under Cross Section Dependence, Econometrics Journal 6, 217-259.

[23] Sul, D. (2006), Panel Unit Root Tests Under Cross Section Dependence with Recursive Mean Adjustment, mimeo, University of Auckland. 
Table 1: Finite sample (average) rejection rates for Pesaran's (2005) CADF and CIPS statistics, Moon and Perron's (2004) $t_{a}^{*}$ and $t_{b}^{*}$ statistics, Bai and Ng's (2004a) $A D F_{\hat{E}}^{c}, P_{\hat{E}}^{c}$, and $A D F_{\hat{F}}^{c}$ statistics, Breitung and Das's (2006) $t_{r o b}$ and $t_{g l s}$ statistics, and Sul's (2006) $t_{f g l s r m a}$ and $t_{c r m a}$ statistics.

\begin{tabular}{|c|c|c|c|c|c|c|c|c|c|c|c|c|c|}
\hline$\frac{\sigma_{\mathrm{f}}^{2}}{\sigma_{\mathrm{e}}^{2}}$ & $\mathbf{N}$ & $\mathbf{T}$ & CADF & CIPS & $t_{a}^{*}$ & $t_{b}^{*}$ & $A D F_{\hat{E}}^{c}$ & $P_{\hat{E}}^{c}$ & $A D F_{\hat{F}}^{c}$ & $t_{r o b}$ & $t_{g l s}$ & $t_{\text {fglsrma }}$ & $t_{\text {crma }}$ \\
\hline 0.5 & 20 & 20 & 0.28 & 0.53 & 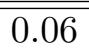 & 0.15 & 0.16 & 0.64 & 0.38 & 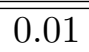 & - & - & - \\
\hline 0.5 & 20 & 50 & 0.12 & 0.16 & 0.07 & 0.13 & 0.07 & 0.15 & 0.14 & 0.07 & 0.04 & 0.07 & 0.52 \\
\hline 0.5 & 20 & 100 & 0.07 & 0.07 & 0.08 & 0.15 & 0.06 & 0.12 & 0.08 & 0.07 & 0.04 & 0.05 & 0.55 \\
\hline 0.5 & 50 & 20 & 0.28 & 0.59 & 0.06 & 0.12 & 0.16 & 0.86 & 0.37 & 0.00 & - & - & - \\
\hline 0.5 & 50 & 50 & 0.12 & 0.16 & 0.04 & 0.10 & 0.07 & 0.21 & 0.14 & 0.03 & - & - & - \\
\hline 0.5 & 50 & 100 & 0.07 & 0.07 & 0.05 & 0.10 & 0.06 & 0.15 & 0.08 & 0.07 & 0.00 & 0.01 & 0.50 \\
\hline 0.5 & 100 & 20 & 0.28 & 0.64 & 0.03 & 0.07 & 0.16 & 0.96 & 0.35 & 0.00 & - & - & - \\
\hline 0.5 & 100 & 50 & 0.12 & 0.16 & 0.04 & 0.07 & 0.07 & 0.29 & 0.13 & 0.00 & - & - & - \\
\hline 0.5 & 100 & 100 & 0.07 & 0.05 & 0.05 & 0.07 & 0.06 & 0.17 & 0.09 & 0.04 & - & - & - \\
\hline$\overline{11}$ & 20 & 20 & 0.28 & 0.52 & 0.09 & 0.17 & 0.16 & 0.64 & 0.37 & 0.02 & - & - & - \\
\hline 1 & 20 & 50 & 0.12 & 0.16 & 0.08 & 0.14 & 0.07 & 0.15 & 0.15 & 0.08 & 0.05 & 0.08 & 0.50 \\
\hline 1 & 20 & 100 & 0.07 & 0.07 & 0.09 & 0.15 & 0.06 & 0.12 & 0.08 & 0.09 & 0.05 & 0.05 & 0.55 \\
\hline 1 & 50 & 20 & 0.28 & 0.59 & 0.11 & 0.18 & 0.16 & 0.86 & 0.37 & 0.00 & - & - & - \\
\hline 1 & 50 & 50 & 0.12 & 0.16 & 0.06 & 0.12 & 0.07 & 0.20 & 0.14 & 0.03 & - & - & - \\
\hline 1 & 50 & 100 & 0.07 & 0.07 & 0.06 & 0.12 & 0.06 & 0.14 & 0.07 & 0.08 & 0.00 & 0.01 & 0.50 \\
\hline 1 & 100 & 20 & 0.28 & 0.64 & 0.09 & 0.13 & 0.16 & 0.96 & 0.35 & 0.00 & - & - & - \\
\hline 1 & 100 & 50 & 0.12 & 0.16 & 0.05 & 0.08 & 0.07 & 0.28 & 0.13 & 0.00 & - & - & - \\
\hline 1 & 100 & 100 & 0.07 & 0.05 & 0.05 & 0.08 & 0.06 & 0.18 & 0.09 & 0.03 & - & - & - \\
\hline$\overline{22}$ & 20 & 20 & 0.28 & 0.53 & 0.14 & 0.22 & 0.16 & 0.64 & 0.38 & 0.04 & 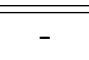 & 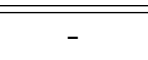 & $\overline{-1}$ \\
\hline 2 & 20 & 50 & 0.12 & 0.15 & 0.09 & 0.16 & 0.07 & 0.15 & 0.14 & 0.09 & 0.06 & 0.10 & 0.51 \\
\hline 2 & 20 & 100 & 0.07 & 0.08 & 0.09 & 0.16 & 0.06 & 0.12 & 0.08 & 0.10 & 0.06 & 0.07 & 0.53 \\
\hline 2 & 50 & 20 & 0.28 & 0.59 & 0.22 & 0.28 & 0.16 & 0.86 & 0.38 & 0.01 & - & - & - \\
\hline 2 & 50 & 50 & 0.12 & 0.15 & 0.09 & 0.14 & 0.07 & 0.21 & 0.15 & 0.03 & - & - & - \\
\hline 2 & 50 & 100 & 0.07 & 0.07 & 0.08 & 0.13 & 0.06 & 0.14 & 0.07 & 0.08 & 0.01 & 0.03 & 0.50 \\
\hline 2 & 100 & 20 & 0.28 & 0.64 & 0.21 & 0.25 & 0.16 & 0.96 & 0.35 & 0.00 & - & - & - \\
\hline 2 & 100 & 50 & 0.12 & 0.16 & 0.08 & 0.13 & 0.07 & 0.28 & 0.13 & 0.00 & - & - & - \\
\hline 2 & 100 & 100 & 0.07 & 0.05 & 0.06 & 0.10 & 0.06 & 0.18 & 0.09 & 0.02 & - & - & - \\
\hline
\end{tabular}

The single common factor is I(1) and idiosyncratic components are I(1). Rejection probabilities are based on 5\% cutoff values from Pesaran (2005), Tables $1 b$ and 3b, 5\% cutoff values of the standard normal distribution, or 5\% Dickey-Fuller critical values for the test statistics as specified in the text. 
Table 2: Finite sample (average) rejection rates for Pesaran's (2005) CADF and CIPS statistics, Moon and Perron's (2004) $t_{a}^{*}$ and $t_{b}^{*}$ statistics, Bai and Ng's (2004a) $A D F_{\hat{E}}^{c}, P_{\hat{E}}^{c}$, and $A D F_{\hat{F}}^{c}$ statistics, Breitung and Das's (2006) $t_{r o b}$ and $t_{g l s}$ statistics, and Sul's (2006) $t_{f g l s r m a}$ and $t_{c r m a}$ statistics.

\begin{tabular}{|c|c|c|c|c|c|c|c|c|c|c|c|c|c|}
\hline$\frac{\sigma_{\mathrm{f}}^{2}}{\sigma_{\mathrm{e}}^{2}}$ & $\mathbf{N}$ & $\mathbf{T}$ & CADF & CIPS & $t_{a}^{*}$ & $t_{b}^{*}$ & $A D F_{\hat{E}}^{c}$ & $P_{\hat{E}}^{c}$ & $A D F_{\hat{F}}^{c}$ & $t_{r o b}$ & $t_{g l s}$ & $t_{f g l s r m a}$ & $t_{c r m a}$ \\
\hline$\overline{0.5}$ & 20 & 20 & 0.32 & 0.68 & 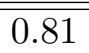 & 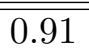 & 0.23 & $\overline{0.94}$ & 0.41 & 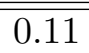 & - & - & - \\
\hline 0.5 & 20 & 50 & 0.17 & 0.54 & 0.99 & 1.00 & 0.25 & 1.00 & 0.15 & 0.38 & 0.68 & 0.60 & 0.60 \\
\hline 0.5 & 20 & 100 & 0.18 & 0.85 & 1.00 & 1.00 & 0.44 & 1.00 & 0.07 & 0.35 & 0.85 & 0.87 & 0.66 \\
\hline 0.5 & 50 & 20 & 0.31 & 0.78 & 0.97 & 0.99 & 0.23 & 1.00 & 0.40 & 0.13 & - & - & - \\
\hline 0.5 & 50 & 50 & 0.18 & 0.71 & 1.00 & 1.00 & 0.26 & 1.00 & 0.13 & 0.40 & - & - & - \\
\hline 0.5 & 50 & 100 & 0.19 & 0.98 & 1.00 & 1.00 & 0.46 & 1.00 & 0.09 & 0.53 & 0.93 & 0.90 & 0.62 \\
\hline 0.5 & 100 & 20 & 0.32 & 0.86 & 0.98 & 0.99 & 0.24 & 1.00 & 0.38 & 0.12 & - & - & - \\
\hline 0.5 & 100 & 50 & 0.19 & 0.80 & 1.00 & 1.00 & 0.27 & 1.00 & 0.14 & 0.30 & - & - & - \\
\hline 0.5 & 100 & 100 & 0.21 & 1.00 & 1.00 & 1.00 & 0.48 & 1.00 & 0.09 & 0.39 & - & - & - \\
\hline 1 & 20 & 20 & 0.32 & 0.68 & 0.82 & 0.92 & 0.23 & 0.94 & 0.40 & 0.09 & - & - & - \\
\hline 1 & 20 & 50 & 0.17 & 0.55 & 0.99 & 1.00 & 0.25 & 1.00 & 0.14 & 0.28 & 0.66 & 0.57 & 0.58 \\
\hline 1 & 20 & 100 & 0.18 & 0.85 & 1.00 & 1.00 & 0.44 & 1.00 & 0.07 & 0.25 & 0.83 & 0.84 & 0.61 \\
\hline 1 & 50 & 20 & 0.31 & 0.79 & 0.96 & 0.98 & 0.23 & 1.00 & 0.38 & 0.10 & - & - & - \\
\hline 1 & 50 & 50 & 0.18 & 0.71 & 1.00 & 1.00 & 0.26 & 1.00 & 0.13 & 0.26 & - & - & - \\
\hline 1 & 50 & 100 & 0.19 & 0.98 & 1.00 & 1.00 & 0.46 & 1.00 & 0.09 & 0.37 & 0.91 & 0.86 & 0.59 \\
\hline 1 & 100 & 20 & 0.32 & 0.86 & 0.97 & 0.98 & 0.24 & 1.00 & 0.38 & 0.08 & - & - & - \\
\hline 1 & 100 & 50 & 0.19 & 0.81 & 1.00 & 1.00 & 0.27 & 1.00 & 0.14 & 0.17 & - & - & - \\
\hline 1 & 100 & 100 & 0.21 & 1.00 & 1.00 & 1.00 & 0.48 & 1.00 & 0.09 & 0.22 & - & - & - \\
\hline 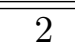 & 20 & 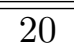 & $\bar{~} 0.32$ & 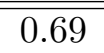 & 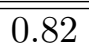 & 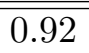 & $\overline{\overline{0.23}}$ & 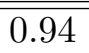 & 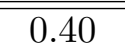 & 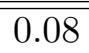 & - & - & - \\
\hline 2 & 20 & 50 & 0.17 & 0.55 & 1.00 & 1.00 & 0.25 & 1.00 & 0.15 & 0.19 & 0.65 & 0.57 & 0.56 \\
\hline 2 & 20 & 100 & 0.18 & 0.86 & 1.00 & 1.00 & 0.44 & 1.00 & 0.08 & 0.19 & 0.82 & 0.83 & 0.59 \\
\hline 2 & 50 & 20 & 0.31 & 0.78 & 0.96 & 0.98 & 0.24 & 1.00 & 0.37 & 0.08 & - & - & - \\
\hline 2 & 50 & 50 & 0.18 & 0.71 & 1.00 & 1.00 & 0.26 & 1.00 & 0.13 & 0.18 & - & - & - \\
\hline 2 & 50 & 100 & 0.19 & 0.98 & 1.00 & 1.00 & 0.46 & 1.00 & 0.10 & 0.24 & 0.89 & 0.83 & 0.57 \\
\hline 2 & 100 & 20 & 0.32 & 0.86 & 0.97 & 0.98 & 0.24 & 1.00 & 0.37 & 0.05 & - & - & - \\
\hline 2 & 100 & 50 & 0.19 & 0.81 & 1.00 & 1.00 & 0.27 & 1.00 & 0.13 & 0.09 & - & - & - \\
\hline 2 & 100 & 100 & 0.21 & 1.00 & 1.00 & 1.00 & 0.48 & 1.00 & 0.10 & 0.11 & - & - & - \\
\hline
\end{tabular}

The single common factor is I(1) and idiosyncratic components are I(0). Rejection probabilities are based on 5\% cutoff values from Pesaran (2005), Tables $1 b$ and 3b, 5\% cutoff values of the standard normal distribution, or 5\% Dickey-Fuller critical values for the test statistics as specified in the text. 
Table 3: Finite sample (average) rejection rates for Pesaran's (2005) CADF and CIPS statistics, Moon and Perron's (2004) $t_{a}^{*}$ and $t_{b}^{*}$ statistics, Bai and Ng's (2004a) $A D F_{\hat{E}}^{c}, P_{\hat{E}}^{c}$, and $A D F_{\hat{F}}^{c}$ statistics, Breitung and Das's (2006) $t_{r o b}$ and $t_{g l s}$ statistics, and Sul's (2006) $t_{f g l s r m a}$ and $t_{c r m a}$ statistics.

\begin{tabular}{|c|c|c|c|c|c|c|c|c|c|c|c|c|c|}
\hline$\frac{\sigma_{\mathrm{f}}^{2}}{\sigma_{\mathrm{e}}^{2}}$ & $\mathbf{N}$ & $\mathbf{T}$ & CADF & CIPS & $t_{a}^{*}$ & $t_{b}^{*}$ & $A D F_{\hat{E}}^{c}$ & $P_{\hat{E}}^{c}$ & $A D F_{\hat{F}}^{c}$ & $t_{r o b}$ & $t_{g l s}$ & $t_{f g l s r m a}$ & $t_{c r m a}$ \\
\hline$\overline{0.5}$ & 20 & 20 & 0.27 & 0.47 & $\bar{~} 0.08$ & $\bar{~} 0.16$ & 0.16 & 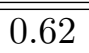 & 0.40 & 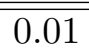 & - & - & - \\
\hline 0.5 & 20 & 50 & 0.11 & 0.10 & 0.09 & 0.14 & 0.07 & 0.12 & 0.18 & 0.15 & 0.06 & 0.10 & 0.49 \\
\hline 0.5 & 20 & 100 & 0.06 & 0.02 & 0.10 & 0.17 & 0.06 & 0.12 & 0.19 & 0.25 & 0.06 & 0.07 & 0.51 \\
\hline 0.5 & 50 & 20 & 0.27 & 0.55 & 0.07 & 0.14 & 0.16 & 0.84 & 0.40 & 0.00 & - & - & - \\
\hline 0.5 & 50 & 50 & 0.11 & 0.08 & 0.06 & 0.11 & 0.07 & 0.20 & 0.17 & 0.08 & - & - & - \\
\hline 0.5 & 50 & 100 & 0.05 & 0.01 & 0.06 & 0.11 & 0.06 & 0.12 & 0.19 & 0.19 & 0.00 & 0.01 & 0.53 \\
\hline 0.5 & 100 & 20 & 0.27 & 0.59 & 0.04 & 0.09 & 0.16 & 0.95 & 0.41 & 0.00 & - & - & - \\
\hline 0.5 & 100 & 50 & 0.11 & 0.06 & 0.05 & 0.08 & 0.07 & 0.25 & 0.16 & 0.00 & - & - & - \\
\hline 0.5 & 100 & 100 & 0.06 & 0.01 & 0.05 & 0.08 & 0.06 & 0.15 & 0.18 & 0.14 & - & - & - \\
\hline 1 & 20 & 20 & 0.27 & 0.47 & 0.13 & 0.21 & 0.16 & 0.61 & 0.41 & 0.02 & - & - & - \\
\hline 1 & 20 & 50 & 0.11 & 0.10 & 0.11 & 0.17 & 0.07 & 0.12 & 0.19 & 0.20 & 0.07 & 0.10 & 0.49 \\
\hline 1 & 20 & 100 & 0.06 & 0.02 & 0.12 & 0.19 & 0.06 & 0.12 & 0.19 & 0.36 & 0.07 & 0.09 & 0.51 \\
\hline 1 & 50 & 20 & 0.27 & 0.55 & 0.17 & 0.24 & 0.16 & 0.84 & 0.40 & 0.00 & - & - & - \\
\hline 1 & 50 & 50 & 0.11 & 0.08 & 0.09 & 0.15 & 0.07 & 0.20 & 0.17 & 0.09 & - & - & - \\
\hline 1 & 50 & 100 & 0.05 & 0.01 & 0.08 & 0.14 & 0.06 & 0.12 & 0.19 & 0.28 & 0.01 & 0.02 & 0.52 \\
\hline 1 & 100 & 20 & 0.27 & 0.58 & 0.13 & 0.19 & 0.16 & 0.95 & 0.40 & 0.00 & - & - & - \\
\hline 1 & 100 & 50 & 0.11 & 0.06 & 0.08 & 0.11 & 0.07 & 0.25 & 0.16 & 0.00 & - & - & - \\
\hline 1 & 100 & 100 & 0.06 & 0.01 & 0.07 & 0.10 & 0.06 & 0.15 & 0.19 & 0.13 & - & - & - \\
\hline 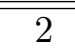 & 20 & 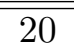 & $\bar{~} 0.27$ & 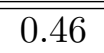 & 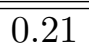 & $\overline{c 0.27}$ & $\overline{\overline{0.16}}$ & 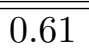 & 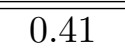 & 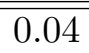 & - & - & - \\
\hline 2 & 20 & 50 & 0.12 & 0.10 & 0.16 & 0.21 & 0.07 & 0.12 & 0.18 & 0.23 & 0.08 & 0.12 & 0.48 \\
\hline 2 & 20 & 100 & 0.06 & 0.02 & 0.17 & 0.24 & 0.06 & 0.12 & 0.18 & 0.44 & 0.08 & 0.12 & 0.50 \\
\hline 2 & 50 & 20 & 0.27 & 0.55 & 0.31 & 0.36 & 0.16 & 0.84 & 0.40 & 0.01 & - & - & - \\
\hline 2 & 50 & 50 & 0.11 & 0.08 & 0.18 & 0.23 & 0.07 & 0.19 & 0.16 & 0.09 & - & - & - \\
\hline 2 & 50 & 100 & 0.05 & 0.01 & 0.16 & 0.21 & 0.06 & 0.12 & 0.20 & 0.35 & 0.01 & 0.05 & 0.51 \\
\hline 2 & 100 & 20 & 0.27 & 0.57 & 0.31 & 0.35 & 0.16 & 0.95 & 0.40 & 0.00 & - & - & - \\
\hline 2 & 100 & 50 & 0.11 & 0.06 & 0.18 & 0.22 & 0.07 & 0.25 & 0.16 & 0.01 & - & - & - \\
\hline 2 & 100 & 100 & 0.06 & 0.00 & 0.13 & 0.18 & 0.06 & 0.14 & 0.19 & 0.12 & - & - & - \\
\hline
\end{tabular}

The single common factor is I(0) and idiosyncratic components are I(1). Rejection probabilities are based on 5\% cutoff values from Pesaran (2005), Tables $1 b$ and 3b, 5\% cutoff values of the standard normal distribution, or 5\% Dickey-Fuller critical values for the test statistics as specified in the text. 
Table 4: Finite sample (average) rejection rates for Pesaran's (2005) CADF and CIPS statistics, Moon and Perron's (2004) $t_{a}^{*}$ and $t_{b}^{*}$ statistics, Bai and Ng's (2004a) $A D F_{\hat{E}}^{c}, P_{\hat{E}}^{c}$, and $A D F_{\hat{F}}^{c}$ statistics, Breitung and Das's (2006) $t_{r o b}$ and $t_{g l s}$ statistics, and Sul's (2006) $t_{f g l s r m a}$ and $t_{c r m a}$ statistics.

\begin{tabular}{|c|c|c|c|c|c|c|c|c|c|c|c|c|c|}
\hline$\frac{\sigma_{\mathrm{f}}^{2}}{\sigma_{\mathrm{e}}^{2}}$ & $\mathbf{N}$ & $\mathbf{T}$ & CADF & CIPS & $t_{a}^{*}$ & $t_{b}^{*}$ & $A D F_{\hat{E}}^{c}$ & $P_{\hat{E}}^{c}$ & $A D F_{\hat{F}}^{c}$ & $t_{r o b}$ & $t_{g l s}$ & $t_{f g l s r m a}$ & $t_{c r m a}$ \\
\hline$\overline{0.5}$ & 20 & 20 & 0.31 & 0.65 & 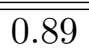 & 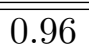 & 0.23 & $\overline{0.94}$ & 0.44 & $\overline{00.15}$ & - & - & - \\
\hline 0.5 & 20 & 50 & 0.16 & 0.48 & 1.00 & 0.99 & 0.26 & 1.00 & 0.18 & 0.72 & 0.88 & 0.79 & 0.61 \\
\hline 0.5 & 20 & 100 & 0.16 & 0.75 & 1.00 & 1.00 & 0.48 & 1.00 & 0.17 & 0.95 & 1.00 & 1.00 & 0.67 \\
\hline 0.5 & 50 & 20 & 0.30 & 0.74 & 0.99 & 1.00 & 0.24 & 1.00 & 0.41 & 0.20 & - & - & - \\
\hline 0.5 & 50 & 50 & 0.17 & 0.58 & 1.00 & 1.00 & 0.27 & 1.00 & 0.17 & 0.78 & - & - & - \\
\hline 0.5 & 50 & 100 & 0.17 & 0.95 & 1.00 & 1.00 & 0.49 & 1.00 & 0.22 & 0.98 & 0.99 & 0.99 & 0.64 \\
\hline 0.5 & 100 & 20 & 0.31 & 0.82 & 1.00 & 1.00 & 0.24 & 1.00 & 0.41 & 0.18 & - & - & - \\
\hline 0.5 & 100 & 50 & 0.17 & 0.69 & 1.00 & 1.00 & 0.29 & 1.00 & 0.19 & 0.62 & - & - & - \\
\hline 0.5 & 100 & 100 & 0.19 & 1.00 & 1.00 & 1.00 & 0.52 & 1.00 & 0.16 & 0.95 & - & - & - \\
\hline 1 & 20 & 20 & 0.31 & 0.64 & 0.91 & 0.96 & 0.23 & 0.94 & 0.43 & 0.13 & - & - & - \\
\hline 1 & 20 & 50 & 0.16 & 0.48 & 1.00 & 1.00 & 0.26 & 1.00 & 0.17 & 0.56 & 0.88 & 0.79 & 0.58 \\
\hline 1 & 20 & 100 & 0.16 & 0.74 & 1.00 & 1.00 & 0.48 & 1.00 & 0.17 & 0.85 & 1.00 & 1.00 & 0.62 \\
\hline 1 & 50 & 20 & 0.30 & 0.73 & 0.99 & 1.00 & 0.24 & 1.00 & 0.41 & 0.16 & - & - & - \\
\hline 1 & 50 & 50 & 0.17 & 0.59 & 1.00 & 1.00 & 0.27 & 1.00 & 0.17 & 0.57 & - & - & - \\
\hline 1 & 50 & 100 & 0.17 & 0.95 & 1.00 & 1.00 & 0.49 & 1.00 & 0.20 & 0.93 & 0.99 & 0.98 & 0.61 \\
\hline 1 & 100 & 20 & 0.31 & 0.82 & 1.00 & 1.00 & 0.24 & 1.00 & 0.41 & 0.12 & - & - & - \\
\hline 1 & 100 & 50 & 0.17 & 0.69 & 1.00 & 1.00 & 0.29 & 1.00 & 0.18 & 0.39 & - & - & - \\
\hline 1 & 100 & 100 & 0.19 & 1.00 & 1.00 & 1.00 & 0.52 & 1.00 & 0.15 & 0.76 & - & - & - \\
\hline 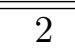 & 20 & 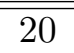 & $\bar{~} 0.31$ & 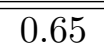 & 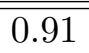 & 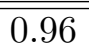 & $\overline{0.23}$ & 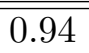 & $\bar{~} 0.43$ & 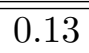 & - & - & - \\
\hline 2 & 20 & 50 & 0.16 & 0.47 & 1.00 & 1.00 & 0.26 & 1.00 & 0.18 & 0.44 & 0.89 & 0.80 & 0.56 \\
\hline 2 & 20 & 100 & 0.16 & 0.75 & 1.00 & 1.00 & 0.48 & 1.00 & 0.17 & 0.73 & 1.00 & 1.00 & 0.58 \\
\hline 2 & 50 & 20 & 0.30 & 0.73 & 0.99 & 1.00 & 0.24 & 1.00 & 0.40 & 0.12 & - & - & - \\
\hline 2 & 50 & 50 & 0.17 & 0.60 & 1.00 & 1.00 & 0.27 & 1.00 & 0.17 & 0.40 & - & - & - \\
\hline 2 & 50 & 100 & 0.17 & 0.95 & 1.00 & 1.00 & 0.49 & 1.00 & 0.20 & 0.80 & 0.99 & 0.98 & 0.58 \\
\hline 2 & 100 & 20 & 0.31 & 0.82 & 1.00 & 1.00 & 0.24 & 1.00 & 0.42 & 0.08 & - & - & - \\
\hline 2 & 100 & 50 & 0.17 & 0.69 & 1.00 & 1.00 & 0.29 & 1.00 & 0.18 & 0.24 & - & - & - \\
\hline 2 & 100 & 100 & 0.19 & 1.00 & 1.00 & 1.00 & 0.52 & 1.00 & 0.16 & 0.55 & - & - & - \\
\hline
\end{tabular}

The single common factor is $I(0)$ and idiosyncratic components are $I(0)$. Rejection probabilities are based on $5 \%$ cutoff values from Pesaran (2005), Tables $1 b$ and 3b, 5\% cutoff values of the standard normal distribution, or 5\% Dickey-Fuller critical values for the test statistics as specified in the text. 
Table 5: Finite sample (average) rejection rates for Pesaran's (2005) CADF and CIPS statistics, Moon and Perron's (2004) $t_{a}^{*}$ and $t_{b}^{*}$ statistics, Bai and Ng's (2004a) $A D F_{\hat{E}}^{c}, P_{\hat{E}}^{c}$, and $A D F_{\hat{F}}^{c}$ statistics, Breitung and Das's (2006) $t_{r o b}$ and $t_{g l s}$ statistics, and Sul's (2006) $t_{f g l s r m a}$ and $t_{c r m a}$ statistics.

\begin{tabular}{|c|c|c|c|c|c|c|c|c|c|c|c|c|c|}
\hline$\frac{\sigma_{\mathrm{f}}^{2}}{\sigma_{\mathrm{e}}^{2}}$ & $\mathbf{N}$ & $\mathbf{T}$ & CADF & CIPS & $t_{a}^{*}$ & $t_{b}^{*}$ & $A D F_{\hat{E}}^{c}$ & $P_{\hat{E}}^{c}$ & $A D F_{\hat{F}}^{c}$ & $t_{r o b}$ & $t_{g l s}$ & $t_{f g l s r m a}$ & $t_{\text {crma }}$ \\
\hline$\overline{0.5}$ & 20 & 20 & 0.30 & 0.63 & 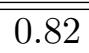 & 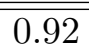 & 0.22 & 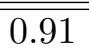 & 0.46 & 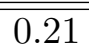 & - & - & - \\
\hline 0.5 & 20 & 50 & 0.16 & 0.38 & 0.98 & 0.99 & 0.24 & 0.99 & 0.25 & 0.83 & 0.86 & 0.77 & 0.59 \\
\hline 0.5 & 20 & 100 & 0.17 & 0.77 & 1.00 & 1.00 & 0.42 & 1.00 & 0.30 & 0.99 & 1.00 & 1.00 & 0.60 \\
\hline 0.5 & 50 & 20 & 0.29 & 0.70 & 0.97 & 0.98 & 0.23 & 0.99 & 0.42 & 0.23 & - & - & - \\
\hline 0.5 & 50 & 50 & 0.16 & 0.54 & 1.00 & 1.00 & 0.24 & 1.00 & 0.21 & 0.82 & - & - & - \\
\hline 0.5 & 50 & 100 & 0.17 & 0.97 & 1.00 & 1.00 & 0.43 & 1.00 & 0.29 & 0.98 & 0.99 & 0.92 & 0.13 \\
\hline 0.5 & 100 & 20 & 0.30 & 0.77 & 0.98 & 0.99 & 0.23 & 1.00 & 0.43 & 0.25 & - & - & - \\
\hline 0.5 & 100 & 50 & 0.16 & 0.59 & 0.99 & 0.99 & 0.25 & 1.00 & 0.26 & 0.78 & - & - & - \\
\hline 0.5 & 100 & 100 & 0.19 & 1.00 & 1.00 & 1.00 & 0.45 & 1.00 & 0.35 & 0.97 & - & - & - \\
\hline 1 & 20 & 20 & 0.30 & 0.60 & 0.78 & 0.85 & 0.22 & 0.90 & 0.45 & 0.18 & - & - & - \\
\hline 1 & 20 & 50 & 0.16 & 0.39 & 0.96 & 0.97 & 0.22 & 0.97 & 0.26 & 0.74 & 0.84 & 0.73 & 0.54 \\
\hline 1 & 20 & 100 & 0.18 & 0.80 & 1.00 & 1.00 & 0.39 & 1.00 & 0.31 & 0.97 & 1.00 & 0.99 & 0.52 \\
\hline 1 & 50 & 20 & 0.29 & 0.70 & 0.94 & 0.96 & 0.23 & 1.00 & 0.41 & 0.19 & - & - & - \\
\hline 1 & 50 & 50 & 0.16 & 0.54 & 0.98 & 0.98 & 0.22 & 1.00 & 0.20 & 0.64 & - & - & - \\
\hline 1 & 50 & 100 & 0.18 & 0.97 & 0.99 & 0.99 & 0.40 & 1.00 & 0.29 & 0.92 & 0.96 & 0.81 & 0.05 \\
\hline 1 & 100 & 20 & 0.30 & 0.77 & 0.92 & 0.94 & 0.23 & 1.00 & 0.42 & 0.17 & - & - & - \\
\hline 1 & 100 & 50 & 0.16 & 0.59 & 0.96 & 0.97 & 0.24 & 1.00 & 0.25 & 0.55 & - & - & - \\
\hline 1 & 100 & 100 & 0.20 & 1.00 & 0.98 & 0.98 & 0.41 & 1.00 & 0.34 & 0.88 & - & - & - \\
\hline $\bar{~} 2$ & 20 & 20 & $\bar{~} 0.30$ & 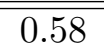 & 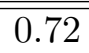 & 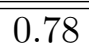 & $\overline{0.21}$ & $\overline{c 0.88}$ & 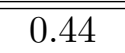 & 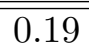 & - & - & - \\
\hline 2 & 20 & 50 & 0.17 & 0.42 & 0.89 & 0.91 & 0.21 & 0.92 & 0.26 & 0.63 & 0.77 & 0.66 & 0.49 \\
\hline 2 & 20 & 100 & 0.21 & 0.85 & 0.98 & 0.98 & 0.35 & 1.00 & 0.31 & 0.93 & 0.99 & 0.94 & 0.41 \\
\hline 2 & 50 & 20 & 0.29 & 0.68 & 0.88 & 0.90 & 0.22 & 0.99 & 0.41 & 0.15 & - & - & - \\
\hline 2 & 50 & 50 & 0.17 & 0.54 & 0.93 & 0.94 & 0.21 & 0.99 & 0.20 & 0.50 & - & - & - \\
\hline 2 & 50 & 100 & 0.19 & 0.97 & 0.97 & 0.97 & 0.36 & 1.00 & 0.29 & 0.81 & 0.90 & 0.65 & 0.01 \\
\hline 2 & 100 & 20 & 0.29 & 0.77 & 0.84 & 0.85 & 0.22 & 1.00 & 0.42 & 0.12 & - & - & - \\
\hline 2 & 100 & 50 & 0.16 & 0.60 & 0.88 & 0.88 & 0.21 & 0.99 & 0.25 & 0.39 & - & - & - \\
\hline 2 & 100 & 100 & 0.21 & 1.00 & 0.92 & 0.92 & 0.36 & 1.00 & 0.34 & 0.72 & - & - & - \\
\hline
\end{tabular}

I(0) data generated by a Pesaran DGP. Rejection probabilities are based on 5\% cutoff values from Pesaran (2005), Tables $1 b$ and $3 b, 5 \%$ cutoff values of the standard normal distribution, or 5\% Dickey-Fuller critical values for the test statistics as specified in the text. 
Table 6: Finite sample (average) rejection rates for Pesaran's (2005) CADF and CIPS statistics, Moon and Perron's (2004) $t_{a}^{*}$ and $t_{b}^{*}$ statistics, Bai and Ng's (2004a) $P_{\hat{E}}^{c}$ statistic, Breitung and Das's (2006) $t_{r o b}$ and $t_{g l s}$ statistics, and Sul's (2006) $t_{f g l s r m a}$ and $t_{c r m a}$ statistics. Proportions of repetitions when Bai and Ng's (2004a) $M Q_{c}^{c}$ and $M Q_{f}^{c}$ statistics chose the correct number of common stochastic trends.

\begin{tabular}{|c|c|c|c|c|c|c|c|c|c|c|c|c|c|}
\hline$\frac{\sigma_{\mathrm{f}}^{2}}{\sigma_{\mathrm{e}}^{2}}$ & $\mathbf{N}$ & $\mathbf{T}$ & CADF & CIPS & $t_{a}^{*}$ & $t_{b}^{*}$ & $P_{\hat{E}}^{c}$ & $M Q_{c}^{c}$ & $M Q_{f}^{c}$ & $t_{r o b}$ & $t_{g l s}$ & $t_{f g l s r m a}$ & $t_{\text {crma }}$ \\
\hline 0.5 & 20 & 20 & 0.31 & 0.54 & 0.09 & 0.18 & 0.36 & 1.00 & 1.00 & 0.02 & - & - & - \\
\hline 0.5 & 20 & 50 & 0.15 & 0.26 & 0.07 & 0.15 & 0.15 & 1.00 & 1.00 & 0.06 & 0.05 & 0.08 & 0.50 \\
\hline 0.5 & 20 & 100 & 0.08 & 0.19 & 0.08 & 0.16 & 0.10 & 1.00 & 1.00 & 0.08 & 0.04 & 0.04 & 0.56 \\
\hline 0.5 & 50 & 20 & 0.30 & 0.60 & 0.09 & 0.15 & 0.51 & 1.00 & 1.00 & 0.00 & - & - & - \\
\hline 0.5 & 50 & 50 & 0.14 & 0.29 & 0.04 & 0.10 & 0.16 & 1.00 & 1.00 & 0.03 & - & - & - \\
\hline 0.5 & 50 & 100 & 0.08 & 0.20 & 0.06 & 0.12 & 0.12 & 1.00 & 1.00 & 0.07 & 0.00 & 0.01 & 0.53 \\
\hline 0.5 & 100 & 20 & 0.30 & 0.61 & 0.07 & 0.14 & 0.67 & 1.00 & 1.00 & 0.00 & - & - & - \\
\hline 0.5 & 100 & 50 & 0.14 & 0.29 & 0.04 & 0.08 & 0.23 & 1.00 & 1.00 & 0.00 & - & - & - \\
\hline 0.5 & 100 & 100 & 0.08 & 0.23 & 0.03 & 0.06 & 0.13 & 1.00 & 1.00 & 0.03 & - & - & - \\
\hline 1 & 20 & 20 & 0.32 & 0.53 & 0.11 & 0.20 & 0.35 & 1.00 & 1.00 & 0.02 & . & - & - \\
\hline 1 & 20 & 50 & 0.15 & 0.30 & 0.08 & 0.15 & 0.14 & 1.00 & 1.00 & 0.08 & 0.05 & 0.09 & 0.50 \\
\hline 1 & 20 & 100 & 0.09 & 0.24 & 0.10 & 0.16 & 0.10 & 1.00 & 1.00 & 0.08 & 0.04 & 0.05 & 0.56 \\
\hline 1 & 50 & 20 & 0.31 & 0.59 & 0.14 & 0.22 & 0.51 & 1.00 & 1.00 & 0.00 & - & - & - \\
\hline 1 & 50 & 50 & 0.15 & 0.34 & 0.05 & 0.11 & 0.16 & 1.00 & 1.00 & 0.04 & - & - & - \\
\hline 1 & 50 & 100 & 0.08 & 0.25 & 0.06 & 0.13 & 0.12 & 1.00 & 1.00 & 0.08 & 0.01 & 0.02 & 0.53 \\
\hline 1 & 100 & 20 & 0.31 & 0.60 & 0.12 & 0.20 & 0.67 & 1.00 & 1.00 & 0.00 & 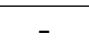 & - & - \\
\hline 1 & 100 & 50 & 0.14 & 0.33 & 0.06 & 0.10 & 0.22 & 1.00 & 1.00 & 0.00 & - & - & - \\
\hline 1 & 100 & 100 & 0.08 & 0.28 & 0.04 & 0.07 & 0.13 & 1.00 & 1.00 & 0.02 & - & - & - \\
\hline 2 & 20 & 20 & 0.33 & 0.55 & 0.16 & 0.24 & 0.35 & 1.00 & $\overline{c 1.00}$ & 0.04 & 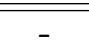 & - & - \\
\hline 2 & 20 & 50 & 0.15 & 0.34 & 0.09 & 0.16 & 0.15 & 1.00 & 1.00 & 0.08 & 0.06 & 0.09 & 0.48 \\
\hline 2 & 20 & 100 & 0.09 & 0.29 & 0.10 & 0.17 & 0.11 & 1.00 & 1.00 & 0.09 & 0.06 & 0.06 & 0.55 \\
\hline 2 & 50 & 20 & 0.33 & 0.60 & 0.20 & 0.27 & 0.51 & 1.00 & 1.00 & 0.02 & - & - & - \\
\hline 2 & 50 & 50 & 0.15 & 0.38 & 0.07 & 0.13 & 0.16 & 1.00 & 1.00 & 0.03 & - & - & - \\
\hline 2 & 50 & 100 & 0.08 & 0.30 & 0.07 & 0.14 & 0.12 & 1.00 & 1.00 & 0.09 & 0.01 & 0.03 & 0.52 \\
\hline 2 & 100 & 20 & 0.33 & 0.60 & 0.19 & 0.26 & 0.68 & 1.00 & 1.00 & 0.00 & - & - & - \\
\hline 2 & 100 & 50 & 0.14 & 0.37 & 0.10 & 0.13 & 0.23 & 1.00 & 1.00 & 0.01 & - & - & - \\
\hline 2 & 100 & 100 & 0.09 & 0.34 & 0.05 & 0.08 & 0.12 & 1.00 & 1.00 & 0.02 & - & - & - \\
\hline
\end{tabular}

The data contains 2 common factors. Common factors are I(1) and idiosyncratic components are I(1). Rejection probabilities are based on 5\% cutoff values from Pesaran (2005), Tables $1 \mathrm{~b}$ and $36,5 \%$ cutoff values of the standard normal distribution, or 5\% Dickey-Fuller critical values for the test statistics as specified in the text. 
Table 7: Finite sample (average) rejection rates for Pesaran's (2005) CADF and CIPS statistics, Moon and Perron's (2004) $t_{a}^{*}$ and $t_{b}^{*}$ statistics, Bai and Ng's (2004a) $P_{\hat{E}}^{c}$ statistic, Breitung and Das's (2006) $t_{r o b}$ and $t_{g l s}$ statistics, and Sul's (2006) $t_{f g l s r m a}$ and $t_{c r m a}$ statistics. Proportions of repetitions when Bai and Ng's (2004a) $M Q_{c}^{c}$ and $M Q_{f}^{c}$ statistics chose the correct number of common stochastic trends.

\begin{tabular}{|c|c|c|c|c|c|c|c|c|c|c|c|c|c|}
\hline$\frac{\sigma_{\mathrm{f}}^{2}}{\sigma_{\mathrm{e}}^{2}}$ & $\mathbf{N}$ & $T$ & CADF & CIPS & $t_{a}^{*}$ & $t_{b}^{*}$ & $P_{\hat{E}}^{c}$ & $M Q_{c}^{c}$ & $M Q_{f}^{c}$ & $t_{r o b}$ & $t_{g l s}$ & $t_{f g l s r m a}$ & $t_{\text {crma }}$ \\
\hline 0.5 & 20 & 20 & 0.32 & 0.60 & 0.71 & 0.85 & 0.77 & 1.00 & 1.00 & 0.08 & - & - & - \\
\hline 0.5 & 20 & 50 & 0.17 & 0.45 & 0.99 & 1.00 & 0.99 & 1.00 & 1.00 & 0.25 & 0.62 & 0.54 & 0.55 \\
\hline 0.5 & 20 & 100 & 0.14 & 0.54 & 1.00 & 1.00 & 1.00 & 1.00 & 1.00 & 0.28 & 0.85 & 0.85 & 0.63 \\
\hline 0.5 & 50 & 20 & 0.33 & 0.67 & 0.88 & 0.94 & 0.98 & 1.00 & 1.00 & 0.08 & - & - & - \\
\hline 0.5 & 50 & 50 & 0.17 & 0.54 & 1.00 & 1.00 & 1.00 & 1.00 & 1.00 & 0.21 & - & - & - \\
\hline 0.5 & 50 & 100 & 0.14 & 0.60 & 1.00 & 1.00 & 1.00 & 1.00 & 1.00 & 0.28 & 0.86 & 0.85 & 0.55 \\
\hline 0.5 & 100 & 20 & 0.33 & 0.71 & 0.96 & 0.98 & 1.00 & 1.00 & 1.00 & 0.09 & - & - & - \\
\hline 0.5 & 100 & 50 & 0.17 & 0.54 & 1.00 & 1.00 & 1.00 & 1.00 & 1.00 & 0.17 & - & - & - \\
\hline 0.5 & 100 & 100 & 0.13 & 0.61 & 1.00 & 1.00 & 1.00 & 1.00 & 1.00 & 0.27 & - & - & - \\
\hline 1 & 20 & 20 & 0.33 & 0.59 & 0.71 & 0.85 & 0.78 & 1.00 & 1.00 & 0.07 & - & - & - \\
\hline 1 & 20 & 50 & 0.16 & 0.44 & 1.00 & 1.00 & 0.99 & 1.00 & 1.00 & 0.18 & 0.58 & 0.54 & 0.51 \\
\hline 1 & 20 & 100 & 0.13 & 0.48 & 1.00 & 1.00 & 1.00 & 1.00 & 1.00 & 0.18 & 0.83 & 0.83 & 0.60 \\
\hline 1 & 50 & 20 & 0.33 & 0.63 & 0.88 & 0.93 & 0.98 & 1.00 & 1.00 & 0.05 & - & - & - \\
\hline 1 & 50 & 50 & 0.17 & 0.51 & 1.00 & 1.00 & 1.00 & 1.00 & 1.00 & 0.14 & - & - & - \\
\hline 1 & 50 & 100 & 0.12 & 0.55 & 1.00 & 1.00 & 1.00 & 1.00 & 1.00 & 0.20 & 0.82 & 0.82 & 0.53 \\
\hline 1 & 100 & 20 & 0.34 & 0.68 & 0.94 & 0.97 & 1.00 & 1.00 & 1.00 & 0.06 & - & - & - \\
\hline 1 & 100 & 50 & 0.16 & 0.51 & 1.00 & 1.00 & 1.00 & 1.00 & 1.00 & 0.09 & - & - & - \\
\hline 1 & 100 & 100 & 0.12 & 0.54 & 1.00 & 1.00 & 1.00 & 1.00 & 1.00 & 0.14 & - & - & - \\
\hline 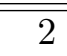 & 20 & 20 & 0.34 & 0.57 & $\overline{c 0.71}$ & $\overline{c 0.84}$ & 0.79 & 1.00 & $\begin{array}{l}1.00 \\
\end{array}$ & 0.05 & - & - & - \\
\hline 2 & 20 & 50 & 0.16 & 0.44 & 1.00 & 1.00 & 0.99 & 1.00 & 1.00 & 0.15 & 0.58 & 0.54 & 0.51 \\
\hline 2 & 20 & 100 & 0.12 & 0.45 & 1.00 & 1.00 & 1.00 & 1.00 & 1.00 & 0.14 & 0.83 & 0.83 & 0.57 \\
\hline 2 & 50 & 20 & 0.34 & 0.61 & 0.87 & 0.93 & 0.99 & 1.00 & 1.00 & 0.04 & - & - & - \\
\hline 2 & 50 & 50 & 0.17 & 0.50 & 1.00 & 1.00 & 1.00 & 1.00 & 1.00 & 0.09 & - & - & - \\
\hline 2 & 50 & 100 & 0.12 & 0.50 & 1.00 & 1.00 & 1.00 & 1.00 & 1.00 & 0.14 & 0.79 & 0.80 & 0.52 \\
\hline 2 & 100 & 20 & 0.35 & 0.65 & 0.94 & 0.97 & 1.00 & 1.00 & 1.00 & 0.04 & - & - & - \\
\hline 2 & 100 & 50 & 0.16 & 0.50 & 1.00 & 1.00 & 1.00 & 1.00 & 1.00 & 0.05 & - & - & - \\
\hline 2 & 100 & 100 & 0.11 & 0.48 & 1.00 & 1.00 & 1.00 & 1.00 & 1.00 & 0.09 & - & - & - \\
\hline
\end{tabular}

The data contains 2 common factors. Common factors are I(1) and idiosyncratic components are I(0). Rejection probabilities are based on 5\% cutoff values from Pesaran (2005), Tables $1 \mathrm{~b}$ and $36,5 \%$ cutoff values of the standard normal distribution, or 5\% Dickey-Fuller critical values for the test statistics as specified in the text. 
Table 8: Finite sample (average) rejection rates for Pesaran's (2005) CADF and CIPS statistics, Moon and Perron's (2004) $t_{a}^{*}$ and $t_{b}^{*}$ statistics, Bai and Ng's (2004a) $P_{\hat{E}}^{c}$ statistic, Breitung and Das's (2006) $t_{r o b}$ and $t_{g l s}$ statistics, and Sul's (2006) $t_{f g l s r m a}$ and $t_{c r m a}$ statistics. Proportions of repetitions when Bai and Ng's (2004a) $M Q_{c}^{c}$ and $M Q_{f}^{c}$ statistics chose the correct number of common stochastic trends.

\begin{tabular}{|c|c|c|c|c|c|c|c|c|c|c|c|c|c|}
\hline$\frac{\sigma_{\mathrm{f}}^{2}}{\sigma_{\mathrm{e}}^{2}}$ & $\mathbf{N}$ & $\mathbf{T}$ & CADF & CIPS & $t_{a}^{*}$ & $t_{b}^{*}$ & $P_{\hat{E}}^{c}$ & $M Q_{c}^{c}$ & $M Q_{f}^{c}$ & $t_{r o b}$ & $t_{g l s}$ & $t_{f g l s r m a}$ & $t_{\text {crma }}$ \\
\hline 0.5 & 20 & 20 & 0.31 & 0.53 & $\overline{0.12}$ & $\bar{~} 0.21$ & 0.30 & $\begin{array}{l}0.00 \\
\end{array}$ & 0.00 & $\overline{c 0.03}$ & - & - & - \\
\hline 0.5 & 20 & 50 & 0.14 & 0.20 & 0.09 & 0.17 & 0.12 & 0.00 & 0.00 & 0.17 & 0.07 & 0.11 & 0.51 \\
\hline 0.5 & 20 & 100 & 0.08 & 0.10 & 0.11 & 0.17 & 0.09 & 0.00 & 0.00 & 0.32 & 0.07 & 0.07 & 0.54 \\
\hline 0.5 & 50 & 20 & 0.30 & 0.58 & 0.12 & 0.21 & 0.43 & 0.00 & 0.00 & 0.00 & - & - & - \\
\hline 0.5 & 50 & 50 & 0.13 & 0.19 & 0.06 & 0.13 & 0.12 & 0.00 & 0.00 & 0.08 & - & - & - \\
\hline 0.5 & 50 & 100 & 0.07 & 0.06 & 0.08 & 0.15 & 0.10 & 0.00 & 0.00 & 0.32 & 0.00 & 0.01 & 0.53 \\
\hline 0.5 & 100 & 20 & 0.30 & 0.60 & 0.11 & 0.17 & 0.59 & 0.00 & 0.00 & 0.00 & - & - & - \\
\hline 0.5 & 100 & 50 & 0.13 & 0.23 & 0.07 & 0.10 & 0.17 & 0.00 & 0.00 & 0.00 & - & - & - \\
\hline 0.5 & 100 & 100 & 0.08 & 0.09 & 0.05 & 0.09 & 0.11 & 0.00 & 0.00 & 0.19 & - & - & - \\
\hline 1 & 20 & 20 & 0.32 & 0.54 & 0.16 & 0.26 & 0.30 & 0.00 & 0.00 & 0.05 & 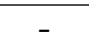 & . & - \\
\hline 1 & 20 & 50 & 0.14 & 0.24 & 0.13 & 0.21 & 0.12 & 0.00 & 0.00 & 0.23 & 0.08 & 0.13 & 0.51 \\
\hline 1 & 20 & 100 & 0.08 & 0.15 & 0.15 & 0.20 & 0.10 & 0.00 & 0.00 & 0.43 & 0.07 & 0.08 & 0.54 \\
\hline 1 & 50 & 20 & 0.31 & 0.59 & 0.23 & 0.33 & 0.42 & 0.00 & 0.00 & 0.01 & - & - & - \\
\hline 1 & 50 & 50 & 0.14 & 0.26 & 0.12 & 0.20 & 0.11 & 0.00 & 0.00 & 0.09 & - & - & - \\
\hline 1 & 50 & 100 & 0.07 & 0.12 & 0.14 & 0.21 & 0.10 & 0.00 & 0.00 & 0.42 & 0.01 & 0.03 & 0.52 \\
\hline 1 & 100 & 20 & 0.31 & 0.59 & 0.25 & 0.32 & 0.59 & 0.00 & 0.00 & 0.00 & - & - & - \\
\hline 1 & 100 & 50 & 0.14 & 0.31 & 0.15 & 0.19 & 0.16 & 0.00 & 0.00 & 0.02 & - & - & - \\
\hline 1 & 100 & 100 & 0.08 & 0.18 & 0.12 & 0.17 & 0.11 & 0.00 & 0.00 & 0.15 & - & - & - \\
\hline 2 & 20 & 20 & 0.34 & 0.57 & 0.24 & 0.33 & $\bar{~} 0.29$ & 0.00 & 0.00 & 0.08 & 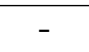 & - & - \\
\hline 2 & 20 & 50 & 0.15 & 0.30 & 0.19 & 0.26 & 0.11 & 0.00 & 0.00 & 0.26 & 0.09 & 0.16 & 0.51 \\
\hline 2 & 20 & 100 & 0.09 & 0.21 & 0.22 & 0.26 & 0.09 & 0.00 & 0.00 & 0.51 & 0.10 & 0.14 & 0.53 \\
\hline 2 & 50 & 20 & 0.33 & 0.60 & 0.33 & 0.41 & 0.42 & 0.00 & 0.00 & 0.03 & - & - & - \\
\hline 2 & 50 & 50 & 0.15 & 0.33 & 0.26 & 0.32 & 0.11 & 0.00 & 0.00 & 0.12 & - & - & - \\
\hline 2 & 50 & 100 & 0.08 & 0.22 & 0.30 & 0.35 & 0.10 & 0.00 & 0.00 & 0.48 & 0.02 & 0.07 & 0.51 \\
\hline 2 & 100 & 20 & 0.33 & 0.60 & 0.37 & 0.44 & 0.60 & 0.00 & 0.00 & 0.01 & - & - & - \\
\hline 2 & 100 & 50 & 0.14 & 0.37 & 0.33 & 0.37 & 0.17 & 0.00 & 0.00 & 0.06 & - & - & - \\
\hline 2 & 100 & 100 & 0.08 & 0.27 & 0.30 & 0.34 & 0.11 & 0.00 & 0.00 & 0.22 & - & - & - \\
\hline
\end{tabular}

The data contains 2 common factors. Common factors are $I(0)$ and idiosyncratic components are I(1). Rejection probabilities are based on 5\% cutoff values from Pesaran (2005), Tables $1 \mathrm{~b}$ and $36,5 \%$ cutoff values of the standard normal distribution, or 5\% Dickey-Fuller critical values for the test statistics as specified in the text. 
Table 9: Finite sample (average) rejection rates for Pesaran's (2005) CADF and CIPS statistics, Moon and Perron's (2004) $t_{a}^{*}$ and $t_{b}^{*}$ statistics, Bai and Ng's (2004a) $P_{\hat{E}}^{c}$ statistic, Breitung and Das's (2006) $t_{r o b}$ and $t_{g l s}$ statistics, and Sul's (2006) $t_{f g l s r m a}$ and $t_{c r m a}$ statistics. Proportions of repetitions when Bai and Ng's (2004a) $M Q_{c}^{c}$ and $M Q_{f}^{c}$ statistics chose the correct number of common stochastic trends.

\begin{tabular}{|c|c|c|c|c|c|c|c|c|c|c|c|c|c|}
\hline$\frac{\sigma_{\mathrm{f}}^{2}}{\sigma_{\mathrm{e}}^{2}}$ & $\mathbf{N}$ & $\mathbf{T}$ & $\mathrm{CADF}$ & CIPS & $t_{a}^{*}$ & $t_{b}^{*}$ & $P_{\hat{E}}^{c}$ & $M Q_{c}^{c}$ & $M Q_{f}^{c}$ & $t_{r o b}$ & $t_{g l s}$ & $t_{f g l s r m a}$ & $t_{c r m o}$ \\
\hline 0.5 & 20 & 20 & 0.32 & 0.59 & 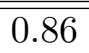 & 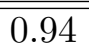 & 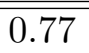 & 0.00 & 0.00 & 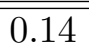 & - & - & - \\
\hline 0.5 & 20 & 50 & 0.17 & 0.46 & 1.00 & 1.00 & 1.00 & 0.00 & 0.00 & 0.65 & 0.92 & 0.80 & 0.57 \\
\hline 0.5 & 20 & 100 & 0.15 & 0.64 & 1.00 & 1.00 & 1.00 & 0.00 & 0.00 & 0.93 & 1.00 & 1.00 & 0.68 \\
\hline 0.5 & 50 & 20 & 0.33 & 0.69 & 0.98 & 0.99 & 0.98 & 0.00 & 0.00 & 0.15 & - & - & - \\
\hline 0.5 & 50 & 50 & 0.18 & 0.56 & 1.00 & 1.00 & 1.00 & 0.00 & 0.00 & 0.60 & - & - & - \\
\hline 0.5 & 50 & 100 & 0.16 & 0.73 & 1.00 & 1.00 & 1.00 & 0.00 & 0.00 & 0.94 & 1.00 & 1.00 & 0.58 \\
\hline 0.5 & 100 & 20 & 0.33 & 0.72 & 1.00 & 1.00 & 1.00 & 0.00 & 0.00 & 0.18 & - & - & - \\
\hline 0.5 & 100 & 50 & 0.17 & 0.55 & 1.00 & 1.00 & 1.00 & 0.00 & 0.00 & 0.58 & - & - & - \\
\hline 0.5 & 100 & 100 & 0.14 & 0.73 & 1.00 & 1.00 & 1.00 & 0.00 & 0.00 & 0.94 & - & - & - \\
\hline 1 & 20 & 20 & 0.33 & 0.59 & 0.86 & 0.96 & 0.79 & 0.00 & 0.00 & 0.13 & - & - & - \\
\hline 1 & 20 & 50 & 0.17 & 0.47 & 1.00 & 1.00 & 1.00 & 0.00 & 0.00 & 0.51 & 0.92 & 0.81 & 0.54 \\
\hline 1 & 20 & 100 & 0.14 & 0.62 & 1.00 & 1.00 & 1.00 & 0.00 & 0.00 & 0.84 & 1.00 & 1.00 & 0.63 \\
\hline 1 & 50 & 20 & 0.34 & 0.67 & 0.98 & 0.99 & 0.98 & 0.00 & 0.00 & 0.11 & - & - & - \\
\hline 1 & 50 & 50 & 0.18 & 0.53 & 1.00 & 1.00 & 1.00 & 0.00 & 0.00 & 0.44 & - & - & - \\
\hline 1 & 50 & 100 & 0.14 & 0.68 & 1.00 & 1.00 & 1.00 & 0.00 & 0.00 & 0.84 & 1.00 & 1.00 & 0.56 \\
\hline 1 & 100 & 20 & 0.35 & 0.69 & 0.99 & 1.00 & 1.00 & 0.00 & 0.00 & 0.13 & - & - & - \\
\hline 1 & 100 & 50 & 0.17 & 0.54 & 1.00 & 1.00 & 1.00 & 0.00 & 0.00 & 0.41 & - & - & - \\
\hline 1 & 100 & 100 & 0.15 & 0.67 & 1.00 & 1.00 & 1.00 & 0.00 & 0.00 & 0.78 & - & - & - \\
\hline 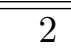 & 20 & 20 & 0.35 & $\overline{0.58}$ & 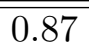 & 0.95 & 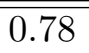 & 0.00 & 0.00 & 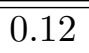 & - & - & - \\
\hline 2 & 20 & 50 & 0.17 & 0.47 & 1.00 & 1.00 & 1.00 & 0.00 & 0.00 & 0.28 & 0.93 & 0.81 & 0.52 \\
\hline 2 & 20 & 100 & 0.14 & 0.59 & 1.00 & 1.00 & 1.00 & 0.00 & 0.00 & 0.43 & 1.00 & 1.00 & 0.59 \\
\hline 2 & 50 & 20 & 0.35 & 0.65 & 0.98 & 0.99 & 0.98 & 0.00 & 0.00 & 0.09 & - & - & - \\
\hline 2 & 50 & 50 & 0.18 & 0.53 & 1.00 & 1.00 & 1.00 & 0.00 & 0.00 & 0.12 & - & - & - \\
\hline 2 & 50 & 100 & 0.14 & 0.63 & 1.00 & 1.00 & 1.00 & 0.00 & 0.00 & 0.29 & 1.00 & 0.99 & 0.54 \\
\hline 2 & 100 & 20 & 0.35 & 0.65 & 0.99 & 1.00 & 1.00 & 0.00 & 0.00 & 0.11 & - & - & - \\
\hline 2 & 100 & 50 & 0.17 & 0.53 & 1.00 & 1.00 & 1.00 & 0.00 & 0.00 & 0.16 & - & - & - \\
\hline 2 & 100 & 100 & 0.13 & 0.63 & 1.00 & 1.00 & 1.00 & 0.00 & 0.00 & 0.29 & - & - & - \\
\hline
\end{tabular}

The data contains 2 common factors. Common factors are $I(0)$ and idiosyncratic components are $I(0)$. Rejection probabilities are based on 5\% cutoff values from Pesaran (2005), Tables $1 \mathrm{~b}$ and $36,5 \%$ cutoff values of the standard normal distribution, or 5\% Dickey-Fuller critical values for the test statistics as specified in the text. 
Table 10: Finite sample (average) rejection rates for Pesaran's (2005) CADF and CIPS statistics, Moon and Perron's (2004) $t_{a}^{*}$ and $t_{b}^{*}$ statistics, Bai and Ng's (2004a) $P_{\hat{E}}^{c}$ statistic, Breitung and Das's (2006) $t_{r o b}$ and $t_{g l s}$ statistics, and Sul's (2006) $t_{f g l s r m a}$ and $t_{c r m a}$ statistics. Proportions of repetitions when Bai and Ng's (2004a) $M Q_{c}^{c}$ and $M Q_{f}^{c}$ statistics chose the correct number of common stochastic trends.

\begin{tabular}{|c|c|c|c|c|c|c|c|c|c|c|c|c|c|}
\hline$\frac{\sigma_{\mathrm{f}}^{2}}{\sigma_{\mathrm{e}}^{2}}$ & $\mathbf{N}$ & $T$ & CADF & CIPS & $t_{a}^{*}$ & $t_{b}^{*}$ & $P_{\hat{E}}^{c}$ & $M Q_{c}^{c}$ & $M Q_{f}^{c}$ & $t_{r o b}$ & $t_{g l s}$ & $t_{f g l s r m a}$ & $t_{\text {crma }}$ \\
\hline 0.5 & 20 & 20 & 0.32 & 0.58 & 0.77 & 0.88 & 0.71 & 0.00 & 0.00 & 0.19 & - & - & - \\
\hline 0.5 & 20 & 50 & 0.17 & 0.44 & 0.96 & 0.98 & 0.94 & 0.00 & 0.00 & 0.78 & 0.86 & 0.74 & 0.45 \\
\hline 0.5 & 20 & 100 & 0.18 & 0.76 & 1.00 & 1.00 & 1.00 & 0.00 & 0.00 & 0.98 & 1.00 & 0.99 & 0.39 \\
\hline 0.5 & 50 & 20 & 0.32 & 0.69 & 0.94 & 0.97 & 0.96 & 0.00 & 0.00 & 0.23 & . & - & - \\
\hline 0.5 & 50 & 50 & 0.18 & 0.57 & 1.00 & 1.00 & 1.00 & 0.00 & 0.00 & 0.80 & - & - & - \\
\hline 0.5 & 50 & 100 & 0.19 & 0.89 & 1.00 & 1.00 & 1.00 & 0.00 & 0.00 & 0.98 & 0.99 & 0.93 & 0.09 \\
\hline 0.5 & 100 & 20 & 0.32 & 0.73 & 0.97 & 0.98 & 1.00 & 0.00 & 0.00 & 0.26 & - & - & - \\
\hline 0.5 & 100 & 50 & 0.17 & 0.57 & 1.00 & 1.00 & 1.00 & 0.00 & 0.00 & 0.76 & - & - & - \\
\hline 0.5 & 100 & 100 & 0.18 & 0.90 & 1.00 & 1.00 & 1.00 & 0.00 & 0.00 & 0.98 & - & - & - \\
\hline 1 & 20 & 20 & 0.33 & 0.57 & 0.69 & 0.78 & 0.65 & 0.00 & 0.00 & 0.18 & . & - & - \\
\hline 1 & 20 & 50 & 0.17 & 0.44 & 0.90 & 0.93 & 0.88 & 0.00 & 0.00 & 0.68 & 0.79 & 0.69 & 0.37 \\
\hline 1 & 20 & 100 & 0.18 & 0.73 & 0.99 & 0.99 & 0.99 & 0.00 & 0.00 & 0.94 & 0.99 & 0.97 & 0.25 \\
\hline 1 & 50 & 20 & 0.33 & 0.68 & 0.88 & 0.92 & 0.92 & 0.00 & 0.00 & 0.17 & - & - & - \\
\hline 1 & 50 & 50 & 0.18 & 0.56 & 0.98 & 0.99 & 1.00 & 0.00 & 0.00 & 0.66 & - & - & - \\
\hline 1 & 50 & 100 & 0.19 & 0.84 & 1.00 & 1.00 & 1.00 & 0.00 & 0.00 & 0.94 & 0.97 & 0.82 & 0.03 \\
\hline 1 & 100 & 20 & 0.33 & 0.69 & 0.91 & 0.92 & 0.98 & 0.00 & 0.00 & 0.20 & - & - & - \\
\hline 1 & 100 & 50 & 0.17 & 0.57 & 0.99 & 0.99 & 1.00 & 0.00 & 0.00 & 0.58 & - & - & - \\
\hline 1 & 100 & 100 & 0.18 & 0.81 & 1.00 & 1.00 & 1.00 & 0.00 & 0.00 & 0.91 & - & - & - \\
\hline 2 & 20 & 20 & 0.34 & 0.58 & 0.59 & 0.67 & 0.59 & 0.00 & $\overline{c 0.00}$ & 0.18 & - & - & $\overline{-1}$ \\
\hline 2 & 20 & 50 & 0.17 & 0.45 & 0.79 & 0.80 & 0.75 & 0.00 & 0.00 & 0.59 & 0.71 & 0.60 & 0.28 \\
\hline 2 & 20 & 100 & 0.19 & 0.71 & 0.95 & 0.95 & 0.95 & 0.00 & 0.00 & 0.90 & 0.96 & 0.90 & 0.16 \\
\hline 2 & 50 & 20 & 0.35 & 0.66 & 0.80 & 0.84 & 0.88 & 0.00 & 0.00 & 0.14 & - & - & - \\
\hline 2 & 50 & 50 & 0.18 & 0.57 & 0.95 & 0.95 & 0.99 & 0.00 & 0.00 & 0.52 & - & - & - \\
\hline 2 & 50 & 100 & 0.20 & 0.80 & 0.99 & 0.99 & 1.00 & 0.00 & 0.00 & 0.89 & 0.92 & 0.62 & 0.01 \\
\hline 2 & 100 & 20 & 0.35 & 0.66 & 0.82 & 0.84 & 0.95 & 0.00 & 0.00 & 0.16 & - & - & - \\
\hline 2 & 100 & 50 & 0.18 & 0.57 & 0.93 & 0.93 & 1.00 & 0.00 & 0.00 & 0.48 & - & - & - \\
\hline 2 & 100 & 100 & 0.19 & 0.81 & 0.98 & 0.98 & 1.00 & 0.00 & 0.00 & 0.81 & - & - & - \\
\hline
\end{tabular}

The data contains 2 common factors. I(0) data generated by a Pesaran DGP. Rejection probabilities are based on $5 \%$ cutoff values from Pesaran (2005), Tables $1 b$ and $3 b, 5 \%$ cutoff values of the standard normal distribution, or $5 \%$ Dickey-Fuller critical values for the test statistics as specified in the text. 
Table 11: Finite sample (average) rejection rates for Moon and Perron's (2004) $t_{a}^{*}$ and $t_{b}^{*}$ statistics, and Bai and Ng's (2004a) $A D F_{\hat{\hat{Q}}}^{c}, P_{\hat{E}}^{c}$, and $A D F_{\hat{\hat{F}}}^{c}$ statistics, the proportions of repetitions when Bai and Ng's (2004a) $M Q_{c}^{c}$ and $M Q_{f}^{c}$ statistics chose the correct number of common stochastic trends, when the number of common factors is misspecified.

\begin{tabular}{|c|c|c|c|c|c|c|c|c|c|c|c|c|}
\hline \multirow[b]{2}{*}{$\frac{\sigma_{\mathrm{f}}^{2}}{\sigma_{\mathrm{e}}^{2}}$} & \multirow[b]{2}{*}{$\mathbf{N}$} & \multirow[b]{2}{*}{$\mathbf{T}$} & \multicolumn{5}{|c|}{$\hat{\mathbf{K}}=\mathbf{1}$} & \multicolumn{5}{|c|}{$\hat{\mathbf{K}}=\mathbf{3}$} \\
\hline & & & $t_{a}^{*}$ & $t_{b}^{*}$ & $A D F_{\hat{E}}^{c}$ & $P_{\hat{E}}^{c}$ & $A D F_{\hat{F}}^{c}$ & $t_{a}^{*}$ & $t_{b}^{*}$ & $P_{\hat{E}}^{c}$ & $M Q_{c}^{c}$ & $M Q_{f}^{c}$ \\
\hline 0.5 & 20 & 20 & 0.20 & 0.17 & 0.16 & 0.62 & 0.40 & 0.18 & 0.31 & 0.61 & 0.00 & 0.01 \\
\hline 0.5 & 20 & 50 & 0.21 & 0.16 & 0.07 & 0.22 & 0.12 & 0.11 & 0.22 & 0.16 & 0.00 & 0.00 \\
\hline 0.5 & 20 & 100 & 0.22 & 0.16 & 0.06 & 0.17 & 0.08 & 0.11 & 0.21 & 0.12 & 0.00 & 0.00 \\
\hline 0.5 & 50 & 20 & 0.22 & 0.2 & 0.16 & 0.79 & 0.39 & 0.29 & 0.37 & 0.83 & 0.00 & 0.01 \\
\hline 0.5 & 50 & 50 & 0.24 & 0.2 & 0.07 & 0.27 & 0.12 & 0.18 & 0.25 & 0.19 & 0.00 & 0.00 \\
\hline 0.5 & 50 & 100 & 0.27 & 0.2 & 0.06 & 0.21 & 0.09 & 0.15 & 0.22 & 0.13 & 0.00 & 0.00 \\
\hline 0.5 & 100 & 20 & 0.32 & 0.2 & 0.16 & 0.86 & 0.38 & 0.29 & 0.34 & 0.92 & 0.00 & 0.01 \\
\hline 0.5 & 100 & 50 & 0.37 & 0.31 & 0.07 & 0.39 & 0.14 & 0.20 & 0.25 & 0.30 & 0.00 & 0.00 \\
\hline 0.5 & 100 & 100 & 0.39 & 0.3 & 0.06 & 0.34 & 0.10 & 0.12 & 0.15 & 0.18 & 0.00 & 0.00 \\
\hline 1 & 20 & 20 & 0.31 & 0.21 & 0.16 & 0.58 & 0.40 & 0.25 & 0.36 & 0.61 & 0.00 & 0.01 \\
\hline 1 & 20 & 50 & 0.32 & 0.16 & 0.07 & 0.25 & 0.12 & 0.18 & 0.29 & 0.16 & 0.00 & 0.00 \\
\hline 1 & 20 & 100 & 0.32 & 0.16 & 0.06 & 0.21 & 0.08 & 0.15 & 0.27 & 0.13 & 0.00 & 0.00 \\
\hline 1 & 50 & 20 & 0.38 & 0.31 & 0.16 & 0.75 & 0.39 & 0.37 & 0.43 & 0.82 & 0.00 & 0.01 \\
\hline 1 & 50 & 50 & 0.38 & 0.2 & 0.07 & 0.32 & 0.13 & 0.25 & 0.34 & 0.19 & 0.00 & 0.00 \\
\hline 1 & 50 & 100 & 0.41 & 0.2 & 0.06 & 0.26 & 0.09 & 0.22 & 0.30 & 0.12 & 0.00 & 0.00 \\
\hline 1 & 100 & 20 & 0.45 & 0.36 & 0.16 & 0.78 & 0.38 & 0.37 & 0.41 & 0.92 & 0.00 & 0.01 \\
\hline 1 & 100 & 50 & 0.48 & 0.38 & 0.07 & 0.42 & 0.14 & 0.30 & 0.34 & 0.30 & 0.00 & 0.00 \\
\hline 1 & 100 & 100 & 0.50 & 0.44 & 0.06 & 0.38 & 0.10 & 0.22 & 0.26 & 0.18 & 0.00 & 0.00 \\
\hline 2 & 20 & 20 & 0.38 & 0.1 & 0.16 & 0.56 & 0.40 & 0.29 & 0.39 & 0.62 & 0.00 & 0.01 \\
\hline 2 & 20 & 50 & 0.41 & 0.1 & 0.07 & 0.29 & 0.12 & 0.23 & 0.34 & 0.16 & 0.00 & 0.00 \\
\hline 2 & 20 & 100 & 0.41 & 0.1 & 0.06 & 0.26 & 0.09 & 0.21 & 0.31 & 0.13 & 0.00 & 0.00 \\
\hline 2 & 50 & 20 & 0.47 & 0.3 & 0.16 & 0.70 & 0.38 & 0.41 & 0.46 & 0.82 & 0.00 & 0.00 \\
\hline 2 & 50 & 50 & 0.47 & 0.2 & 0.07 & 0.33 & 0.13 & 0.32 & 0.38 & 0.20 & 0.00 & 0.00 \\
\hline 2 & 50 & 100 & 0.49 & 0.30 & 0.06 & 0.32 & 0.08 & 0.29 & 0.35 & 0.12 & 0.00 & 0.00 \\
\hline 2 & 100 & 20 & 0.50 & 0.38 & 0.16 & 0.69 & 0.38 & 0.41 & 0.44 & 0.91 & 0.00 & 0.01 \\
\hline 2 & 100 & 50 & 0.52 & 0.3 & 0.07 & 0.42 & 0.14 & 0.35 & 0.38 & 0.30 & 0.00 & 0.00 \\
\hline 2 & 100 & 100 & 0.53 & 0.3 & 0.07 & 0.39 & 0.10 & 0.28 & 0.33 & 0.18 & 0.00 & 0.00 \\
\hline
\end{tabular}

The data contains 2 common factors. Common factors are $I(1)$ and idiosyncratic components are I(1). Rejection probabilities are based on 5\% cutoff values from Pesaran (2005), Tables $1 b$ and 3b, 5\% cutoff values of the standard normal distribution, or 5\% Dickey-Fuller critical values for the test statistics as specified in the text. 
Table 12: Finite sample (average) rejection rates for Moon and Perron's (2004) $t_{a}^{*}$ and $t_{b}^{*}$ statistics, and Bai and Ng's (2004a) $A D F_{\hat{E}}^{c}, P_{\hat{E}}^{c}$, and $A D F_{\hat{F}}^{c}$ statistics, the proportions of repetitions when Bai and Ng's (2004a) $M Q_{c}^{c}$ and $M Q_{f}^{c}$ statistics chose the correct number of common stochastic trends, when the number of common factors is misspecified.

\begin{tabular}{|c|c|c|c|c|c|c|c|c|c|c|c|c|}
\hline \multirow[b]{2}{*}{$\frac{\sigma_{\mathrm{f}}^{2}}{\sigma_{\mathrm{e}}^{2}}$} & \multirow[b]{2}{*}{$\mathbf{N}$} & \multirow[b]{2}{*}{$\mathbf{T}$} & \multicolumn{5}{|c|}{$\hat{\mathbf{K}}=\mathbf{1}$} & \multicolumn{5}{|c|}{$\hat{\mathbf{K}}=\mathbf{3}$} \\
\hline & & & $t_{a}^{*}$ & $t_{b}^{*}$ & $A D F_{\hat{E}}^{c}$ & $P_{\hat{E}}^{c}$ & $A D F_{\hat{F}}^{c}$ & $t_{a}^{*}$ & $t_{b}^{*}$ & $P_{\hat{E}}^{c}$ & $M Q_{c}^{c}$ & $M Q_{f}^{c}$ \\
\hline 0.5 & 20 & 20 & 0.54 & 0.55 & 0.20 & 0.76 & 0.38 & 0.58 & 0.77 & 0.82 & 0.00 & 0.01 \\
\hline 0.5 & 20 & 50 & 0.65 & 0.63 & 0.16 & 0.66 & 0.14 & 0.98 & 1.00 & 0.96 & 0.00 & 0.00 \\
\hline 0.5 & 20 & 100 & 0.71 & 0.69 & 0.21 & 0.73 & 0.08 & 1.00 & 1.00 & 1.00 & 0.00 & 0.00 \\
\hline 0.5 & 50 & 20 & 0.65 & 0.65 & 0.20 & 0.88 & 0.40 & 0.78 & 0.88 & 0.99 & 0.00 & 0.02 \\
\hline 0.5 & 50 & 50 & 0.71 & 0.69 & 0.16 & 0.73 & 0.14 & 1.00 & 1.00 & 1.00 & 0.00 & 0.00 \\
\hline 0.5 & 50 & 100 & 0.75 & 0.74 & 0.22 & 0.81 & 0.10 & 1.00 & 1.00 & 1.00 & 0.00 & 0.00 \\
\hline 0.5 & 100 & 20 & 0.71 & 0.71 & 0.20 & 0.92 & 0.37 & 0.88 & 0.93 & 1.00 & 0.00 & 0.02 \\
\hline 0.5 & 100 & 50 & 0.77 & 0.75 & 0.15 & 0.77 & 0.13 & 1.00 & 1.00 & 1.00 & 0.00 & 0.00 \\
\hline 0.5 & 100 & 100 & 0.80 & 0.79 & 0.21 & 0.84 & 0.08 & 1.00 & 1.00 & 1.00 & 0.00 & 0.00 \\
\hline 1 & 20 & 20 & 0.50 & 0.45 & 0.19 & 0.69 & 0.38 & 0.58 & 0.77 & 0.84 & 0.00 & 0.01 \\
\hline 1 & 20 & 50 & 0.58 & 0.51 & 0.14 & 0.56 & 0.15 & 0.98 & 1.00 & 0.96 & 0.00 & 0.00 \\
\hline 1 & 20 & 100 & 0.63 & 0.55 & 0.18 & 0.61 & 0.08 & 1.00 & 1.00 & 1.00 & 0.00 & 0.00 \\
\hline 1 & 50 & 20 & 0.59 & 0.55 & 0.19 & 0.79 & 0.39 & 0.76 & 0.86 & 0.99 & 0.00 & 0.01 \\
\hline 1 & 50 & 50 & 0.61 & 0.58 & 0.14 & 0.64 & 0.14 & 1.00 & 1.00 & 1.00 & 0.00 & 0.00 \\
\hline 1 & 50 & 100 & 0.66 & 0.61 & 0.19 & 0.70 & 0.09 & 1.00 & 1.00 & 1.00 & 0.00 & 0.00 \\
\hline 1 & 100 & 20 & 0.66 & 0.62 & 0.20 & 0.83 & 0.37 & 0.86 & 0.92 & 1.00 & 0.00 & 0.01 \\
\hline 1 & 100 & 50 & 0.67 & 0.62 & 0.13 & 0.66 & 0.12 & 1.00 & 1.00 & 1.00 & 0.00 & 0.00 \\
\hline 1 & 100 & 100 & 0.71 & 0.67 & 0.18 & 0.73 & 0.08 & 1.00 & 1.00 & 1.00 & 0.00 & 0.00 \\
\hline 2 & 20 & 20 & 0.48 & 0.34 & 0.18 & 0.61 & 0.38 & 0.58 & 0.76 & 0.85 & 0.00 & 0.01 \\
\hline 2 & 20 & 50 & 0.53 & 0.37 & 0.13 & 0.47 & 0.14 & 0.99 & 1.00 & 0.97 & 0.00 & 0.00 \\
\hline 2 & 20 & 100 & 0.56 & 0.41 & 0.15 & 0.52 & 0.08 & 1.00 & 1.00 & 1.00 & 0.00 & 0.00 \\
\hline 2 & 50 & 20 & 0.55 & 0.45 & 0.19 & 0.70 & 0.39 & 0.75 & 0.86 & 0.99 & 0.00 & 0.02 \\
\hline 2 & 50 & 50 & 0.56 & 0.46 & 0.12 & 0.54 & 0.14 & 1.00 & 1.00 & 1.00 & 0.00 & 0.00 \\
\hline 2 & 50 & 100 & 0.60 & 0.47 & 0.15 & 0.58 & 0.09 & 1.00 & 1.00 & 1.00 & 0.00 & 0.00 \\
\hline 2 & 100 & 20 & 0.61 & 0.54 & 0.19 & 0.73 & 0.37 & 0.84 & 0.91 & 1.00 & 0.00 & 0.02 \\
\hline 2 & 100 & 50 & 0.62 & 0.53 & 0.12 & 0.56 & 0.12 & 1.00 & 1.00 & 1.00 & 0.00 & 0.00 \\
\hline 2 & 100 & 100 & 0.65 & 0.56 & 0.15 & 0.64 & 0.08 & 1.00 & 1.00 & 1.00 & 0.00 & 0.00 \\
\hline
\end{tabular}

The data contains 2 common factors. Common factors are $I(1)$ and idiosyncratic components are $I(0)$. Rejection probabilities are based on 5\% cutoff values from Pesaran (2005), Tables $1 b$ and 3b, 5\% cutoff values of the standard normal distribution, or 5\% Dickey-Fuller critical values for the test statistics as specified in the text. 
Table 13: Finite sample (average) rejection rates for Moon and Perron's (2004) $t_{a}^{*}$ and $t_{b}^{*}$ statistics, and Bai and Ng's (2004a) $A D F_{\hat{\hat{Q}}}^{c}, P_{\hat{E}}^{c}$, and $A D F_{\hat{\hat{F}}}^{c}$ statistics, the proportions of repetitions when Bai and Ng's (2004a) $M Q_{c}^{c}$ and $M Q_{f}^{c}$ statistics chose the correct number of common stochastic trends, when the number of common factors is misspecified.

\begin{tabular}{|c|c|c|c|c|c|c|c|c|c|c|c|c|}
\hline \multirow[b]{2}{*}{$\frac{\sigma_{\mathrm{f}}^{2}}{\sigma_{\mathrm{e}}^{2}}$} & \multirow[b]{2}{*}{$\mathbf{N}$} & \multirow[b]{2}{*}{$\mathbf{T}$} & \multicolumn{5}{|c|}{$\hat{\mathbf{K}}=\mathbf{1}$} & \multicolumn{5}{|c|}{$\hat{\mathbf{K}}=\mathbf{3}$} \\
\hline & & & $t_{a}^{*}$ & $t_{b}^{*}$ & $A D F_{\hat{E}}^{c}$ & $P_{\hat{E}}^{c}$ & $A D F_{\hat{F}}^{c}$ & $t_{a}^{*}$ & $t_{b}^{*}$ & $P_{\hat{E}}^{c}$ & $M Q_{c}^{c}$ & $M Q_{f}^{c}$ \\
\hline 0.5 & 20 & 20 & 0.29 & 0.25 & 0.17 & 0.65 & 0.42 & 0.30 & 0.44 & 0.57 & 0.00 & 0.00 \\
\hline 0.5 & 20 & 50 & 0.39 & 0.31 & 0.09 & 0.30 & 0.17 & 0.27 & 0.41 & 0.13 & 0.00 & 0.00 \\
\hline 0.5 & 20 & 100 & 0.49 & 0.40 & 0.08 & 0.33 & 0.16 & 0.27 & 0.39 & 0.10 & 0.00 & 0.00 \\
\hline 0.5 & 50 & 20 & 0.36 & 0.33 & 0.16 & 0.83 & 0.42 & 0.50 & 0.58 & 0.80 & 0.00 & 0.00 \\
\hline 0.5 & 50 & 50 & 0.46 & 0.41 & 0.08 & 0.41 & 0.16 & 0.56 & 0.62 & 0.15 & 0.00 & 0.00 \\
\hline 0.5 & 50 & 100 & 0.60 & 0.55 & 0.08 & 0.45 & 0.18 & 0.56 & 0.63 & 0.10 & 0.00 & 0.00 \\
\hline 0.5 & 100 & 20 & 0.49 & 0.44 & 0.17 & 0.92 & 0.41 & 0.55 & 0.59 & 0.90 & 0.00 & 0.00 \\
\hline 0.5 & 100 & 50 & 0.67 & 0.60 & 0.09 & 0.62 & 0.20 & 0.63 & 0.67 & 0.25 & 0.00 & 0.00 \\
\hline 0.5 & 100 & 100 & 0.81 & 0.75 & 0.09 & 0.68 & 0.20 & 0.68 & 0.74 & 0.15 & 0.00 & 0.00 \\
\hline 1 & 20 & 20 & 0.45 & 0.29 & 0.17 & 0.63 & 0.42 & 0.42 & 0.54 & 0.57 & 0.00 & 0.00 \\
\hline 1 & 20 & 50 & 0.62 & 0.38 & 0.10 & 0.39 & 0.17 & 0.52 & 0.63 & 0.14 & 0.00 & 0.00 \\
\hline 1 & 20 & 100 & 0.77 & 0.52 & 0.10 & 0.48 & 0.15 & 0.59 & 0.69 & 0.10 & 0.00 & 0.00 \\
\hline 1 & 50 & 20 & 0.56 & 0.45 & 0.17 & 0.82 & 0.41 & 0.62 & 0.66 & 0.79 & 0.00 & 0.00 \\
\hline 1 & 50 & 50 & 0.74 & 0.58 & 0.09 & 0.50 & 0.16 & 0.76 & 0.80 & 0.15 & 0.00 & 0.00 \\
\hline 1 & 50 & 100 & 0.89 & 0.78 & 0.09 & 0.63 & 0.18 & 0.89 & 0.92 & 0.11 & 0.00 & 0.00 \\
\hline 1 & 100 & 20 & 0.64 & 0.55 & 0.17 & 0.87 & 0.41 & 0.67 & 0.69 & 0.90 & 0.00 & 0.00 \\
\hline 1 & 100 & 50 & 0.85 & 0.77 & 0.10 & 0.68 & 0.19 & 0.81 & 0.83 & 0.24 & 0.00 & 0.00 \\
\hline 1 & 100 & 100 & 0.97 & 0.92 & 0.10 & 0.82 & 0.20 & 0.94 & 0.94 & 0.14 & 0.00 & 0.00 \\
\hline 2 & 20 & 20 & 0.56 & 0.29 & 0.18 & 0.61 & 0.43 & 0.48 & 0.59 & 0.56 & 0.00 & 0.00 \\
\hline 2 & 20 & 50 & 0.76 & 0.39 & 0.10 & 0.49 & 0.17 & 0.66 & 0.74 & 0.13 & 0.00 & 0.00 \\
\hline 2 & 20 & 100 & 0.94 & 0.58 & 0.12 & 0.65 & 0.16 & 0.82 & 0.87 & 0.10 & 0.00 & 0.00 \\
\hline 2 & 50 & 20 & 0.65 & 0.47 & 0.18 & 0.79 & 0.40 & 0.65 & 0.69 & 0.79 & 0.00 & 0.00 \\
\hline 2 & 50 & 50 & 0.84 & 0.64 & 0.10 & 0.58 & 0.16 & 0.83 & 0.86 & 0.15 & 0.00 & 0.00 \\
\hline 2 & 50 & 100 & 0.97 & 0.88 & 0.11 & 0.77 & 0.17 & 0.95 & 0.95 & 0.11 & 0.00 & 0.00 \\
\hline 2 & 100 & 20 & 0.70 & 0.55 & 0.17 & 0.81 & 0.41 & 0.71 & 0.74 & 0.90 & 0.00 & 0.00 \\
\hline 2 & 100 & 50 & 0.90 & 0.77 & 0.11 & 0.72 & 0.19 & 0.87 & 0.89 & 0.24 & 0.00 & 0.00 \\
\hline 2 & 100 & 100 & 0.99 & 0.95 & 0.13 & 0.89 & 0.19 & 0.97 & 0.97 & 0.14 & 0.00 & 0.00 \\
\hline
\end{tabular}

The data contains 2 common factors. Common factors are $I(0)$ and idiosyncratic components are $I(1)$. Rejection probabilities are based on 5\% cutoff values from Pesaran (2005), Tables $1 b$ and 3b, 5\% cutoff values of the standard normal distribution, or 5\% Dickey-Fuller critical values for the test statistics as specified in the text. 
Table 14: Finite sample (average) rejection rates for Moon and Perron's (2004) $t_{a}^{*}$ and $t_{b}^{*}$ statistics, and Bai and Ng's (2004a) $A D F_{\hat{\hat{Q}}}^{c}, P_{\hat{E}}^{c}$, and $A D F_{\hat{\hat{F}}}^{c}$ statistics, the proportions of repetitions when Bai and Ng's (2004a) $M Q_{c}^{c}$ and $M Q_{f}^{c}$ statistics chose the correct number of common stochastic trends, when the number of common factors is misspecified.

\begin{tabular}{|c|c|c|c|c|c|c|c|c|c|c|c|c|}
\hline & & & \multicolumn{5}{|c|}{$\hat{\mathbf{K}}=\mathbf{1}$} & \multicolumn{5}{|c|}{$\hat{\mathbf{K}}=\mathbf{3}$} \\
\hline$\frac{\sigma_{\mathrm{f}}^{2}}{\sigma_{\mathrm{e}}^{2}}$ & $\mathbf{N}$ & $\mathbf{T}$ & $t_{a}^{*}$ & $t_{b}^{*}$ & $A D F_{\hat{E}}^{c}$ & $P_{\hat{E}}^{c}$ & $A D F_{\hat{F}}^{c}$ & $t_{a}^{*}$ & $t_{b}^{*}$ & $P_{\hat{E}}^{c}$ & $M Q_{c}^{c}$ & $M Q_{f}^{c}$ \\
\hline 0.5 & 20 & 20 & $\begin{array}{l}0.72 \\
\end{array}$ & 0.73 & 0.21 & 0.84 & 0.42 & 0.74 & 0.92 & 0.83 & 0.00 & 0.00 \\
\hline 0.5 & 20 & 50 & 0.96 & 0.96 & 0.23 & 0.93 & 0.17 & 1.00 & 1.00 & 0.99 & 0.00 & 0.00 \\
\hline 0.5 & 20 & 100 & 1.00 & 1.00 & 0.43 & 1.00 & 0.19 & 1.00 & 1.00 & 1.00 & 0.00 & 0.00 \\
\hline 0.5 & 50 & 20 & 0.84 & 0.83 & 0.22 & 0.93 & 0.43 & 0.93 & 0.98 & 0.99 & 0.00 & 0.00 \\
\hline 0.5 & 50 & 50 & 0.99 & 0.99 & 0.23 & 0.98 & 0.17 & 1.00 & 1.00 & 1.00 & 0.00 & 0.00 \\
\hline 0.5 & 50 & 100 & 1.00 & 1.00 & 0.43 & 1.00 & 0.18 & 1.00 & 1.00 & 1.00 & 0.00 & 0.00 \\
\hline 0.5 & 100 & 20 & 0.87 & 0.97 & 0.22 & 0.97 & 0.40 & 0.99 & 0.99 & 1.00 & 0.00 & 0.00 \\
\hline 0.5 & 100 & 50 & 1.00 & 1.00 & 0.22 & 0.98 & 0.16 & 1.00 & 1.00 & 1.00 & 0.00 & 0.00 \\
\hline 0.5 & 100 & 100 & 1.00 & 1.00 & 0.42 & 1.00 & 0.17 & 1.00 & 1.00 & 1.00 & 0.00 & 0.00 \\
\hline $\bar{~} 1$ & 20 & 20 & $\overline{c 0.68}$ & 0.62 & 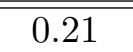 & $\overline{c 0.78}$ & 0.42 & 0.75 & 0.92 & $\bar{~} 0.83$ & 0.00 & 0.00 \\
\hline 1 & 20 & 50 & 0.93 & 0.89 & 0.23 & 0.87 & 0.17 & 1.00 & 1.00 & 0.99 & 0.00 & 0.00 \\
\hline 1 & 20 & 100 & 1.00 & 1.00 & 0.40 & 1.00 & 0.19 & 1.00 & 1.00 & 1.00 & 0.00 & 0.00 \\
\hline 1 & 50 & 20 & 0.77 & 0.72 & 0.22 & 0.88 & 0.43 & 0.93 & 0.97 & 0.99 & 0.00 & 0.00 \\
\hline 1 & 50 & 50 & 0.97 & 0.96 & 0.22 & 0.94 & 0.17 & 1.00 & 1.00 & 1.00 & 0.00 & 0.00 \\
\hline 1 & 50 & 100 & 1.00 & 1.00 & 0.40 & 1.00 & 0.18 & 1.00 & 1.00 & 1.00 & 0.00 & 0.00 \\
\hline 1 & 100 & 20 & 0.81 & 0.78 & 0.21 & 0.90 & 0.41 & 0.98 & 0.99 & 1.00 & 0.00 & 0.00 \\
\hline 1 & 100 & 50 & 0.98 & 0.97 & 0.21 & 0.95 & 0.16 & 1.00 & 1.00 & 1.00 & 0.00 & 0.00 \\
\hline 1 & 100 & 100 & 1.00 & 1.00 & 0.39 & 1.00 & 0.17 & 1.00 & 1.00 & 1.00 & 0.00 & 0.00 \\
\hline 2 & 20 & 20 & 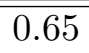 & $\overline{0.50}$ & $\overline{0.21}$ & 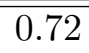 & $\overline{0.42}$ & 0.75 & $\overline{0.92}$ & 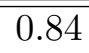 & $\overline{c 0.00}$ & 0.00 \\
\hline 2 & 20 & 50 & 0.91 & 0.76 & 0.22 & 0.80 & 0.17 & 1.00 & 1.00 & 0.99 & 0.00 & 0.00 \\
\hline 2 & 20 & 100 & 0.99 & 0.98 & 0.38 & 0.98 & 0.19 & 1.00 & 1.00 & 1.00 & 0.00 & 0.00 \\
\hline 2 & 50 & 20 & 0.73 & 0.62 & 0.21 & 0.79 & 0.43 & 0.92 & 0.97 & 0.99 & 0.00 & 0.00 \\
\hline 2 & 50 & 50 & 0.95 & 0.87 & 0.21 & 0.88 & 0.17 & 1.00 & 1.00 & 1.00 & 0.00 & 0.00 \\
\hline 2 & 50 & 100 & 1.00 & 0.99 & 0.38 & 1.00 & 0.18 & 1.00 & 1.00 & 1.00 & 0.00 & 0.00 \\
\hline 2 & 100 & 20 & 0.77 & 0.67 & 0.21 & 0.83 & 0.40 & 0.98 & 0.99 & 1.00 & 0.00 & 0.00 \\
\hline 2 & 100 & 50 & 0.96 & 0.93 & 0.20 & 0.90 & 0.16 & 1.00 & 1.00 & 1.00 & 0.00 & 0.00 \\
\hline 2 & 100 & 100 & 1.00 & 1.00 & 0.37 & 1.00 & 0.17 & 1.00 & 1.00 & 1.00 & 0.00 & 0.00 \\
\hline
\end{tabular}

The data contains 2 common factors. Common factors are $I(0)$ and idiosyncratic components are $I(0)$. Rejection probabilities are based on 5\% cutoff values from Pesaran (2005), Tables $1 b$ and 3b, 5\% cutoff values of the standard normal distribution, or 5\% Dickey-Fuller critical values for the test statistics as specified in the text. 
Table 15: Finite sample (average) rejection rates for Moon and Perron's (2004) $t_{a}^{*}$ and $t_{b}^{*}$ statistics, and Bai and Ng's (2004a) $A D F_{\hat{E}}^{c}, P_{\hat{E}}^{c}$, and $A D F_{\hat{F}}^{c}$ statistics, the proportions of repetitions when Bai and Ng's (2004a) $M Q_{c}^{c}$ and $M Q_{f}^{c}$ statistics chose the correct number of common stochastic trends, when the number of common factors is misspecified.

\begin{tabular}{|c|c|c|c|c|c|c|c|c|c|c|c|c|}
\hline & & & \multicolumn{5}{|c|}{$\hat{\mathbf{K}}=\mathbf{1}$} & \multicolumn{5}{|c|}{$\hat{\mathbf{K}}=\mathbf{3}$} \\
\hline$\frac{\sigma_{\mathrm{f}}^{2}}{\sigma_{\mathrm{e}}^{2}}$ & $\mathbf{N}$ & $\mathbf{T}$ & $t_{a}^{*}$ & $t_{b}^{*}$ & $A D F_{\hat{E}}^{c}$ & $P_{\hat{E}}^{c}$ & $A D F_{\hat{F}}^{c}$ & $t_{a}^{*}$ & $t_{b}^{*}$ & $P_{\hat{E}}^{c}$ & $M Q_{c}^{c}$ & $M Q_{f}^{c}$ \\
\hline$\overline{0.5}$ & 20 & 20 & 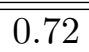 & 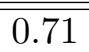 & 0.22 & 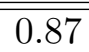 & 0.45 & 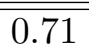 & $\overline{c 0.88}$ & $\bar{~} 0.79$ & 0.00 & 0.00 \\
\hline 0.5 & 20 & 50 & 0.93 & 0.92 & 0.26 & 0.97 & 0.27 & 0.96 & 0.98 & 0.91 & 0.00 & 0.00 \\
\hline 0.5 & 20 & 100 & 0.99 & 0.99 & 0.46 & 1.00 & 0.40 & 1.00 & 1.00 & 1.00 & 0.00 & 0.00 \\
\hline 0.5 & 50 & 20 & 0.88 & 0.88 & 0.23 & 0.97 & 0.46 & 0.91 & 0.96 & 0.97 & 0.00 & 0.00 \\
\hline 0.5 & 50 & 50 & 0.99 & 0.99 & 0.26 & 1.00 & 0.27 & 1.00 & 1.00 & 1.00 & 0.00 & 0.00 \\
\hline 0.5 & 50 & 100 & 1.00 & 1.00 & 0.48 & 1.00 & 0.37 & 1.00 & 1.00 & 1.00 & 0.00 & 0.00 \\
\hline 0.5 & 100 & 20 & 0.92 & 0.92 & 0.23 & 0.99 & 0.42 & 0.95 & 0.97 & 1.00 & 0.00 & 0.00 \\
\hline 0.5 & 100 & 50 & 1.00 & 1.00 & 0.25 & 1.00 & 0.22 & 1.00 & 1.00 & 1.00 & 0.00 & 0.00 \\
\hline 0.5 & 100 & 100 & 1.00 & 1.00 & 0.46 & 1.00 & 0.32 & 1.00 & 1.00 & 1.00 & 0.00 & 0.00 \\
\hline 1 & 20 & 20 & 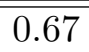 & 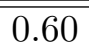 & 0.22 & $\bar{~} 0.83$ & 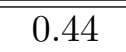 & 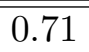 & $\bar{~} 0.84$ & 0.75 & 0.00 & 0.00 \\
\hline 1 & 20 & 50 & 0.89 & 0.81 & 0.25 & 0.94 & 0.27 & 0.93 & 0.96 & 0.84 & 0.00 & 0.00 \\
\hline 1 & 20 & 100 & 0.98 & 0.96 & 0.45 & 1.00 & 0.40 & 1.00 & 1.00 & 0.99 & 0.00 & 0.00 \\
\hline 1 & 50 & 20 & 0.83 & 0.77 & 0.47 & 0.93 & 0.46 & 0.89 & 0.93 & 0.96 & 0.00 & 0.00 \\
\hline 1 & 50 & 50 & 0.99 & 0.98 & 0.27 & 0.99 & 0.27 & 0.99 & 1.00 & 1.00 & 0.00 & 0.00 \\
\hline 1 & 50 & 100 & 1.00 & 1.00 & 0.37 & 1.00 & 0.36 & 1.00 & 1.00 & 1.00 & 0.00 & 0.00 \\
\hline 1 & 100 & 20 & 0.87 & 0.84 & 0.22 & 0.96 & 0.43 & 0.92 & 0.95 & 0.99 & 0.00 & 0.00 \\
\hline 1 & 100 & 50 & 1.00 & 0.99 & 0.24 & 0.99 & 0.22 & 1.00 & 1.00 & 1.00 & 0.00 & 0.00 \\
\hline 1 & 100 & 100 & 1.00 & 1.00 & 0.47 & 1.00 & 0.31 & 1.00 & 1.00 & 1.00 & 0.00 & 0.00 \\
\hline$\overline{\overline{2}}$ & 20 & 20 & "0.62 & "0.45 & 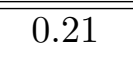 & "0.77 & 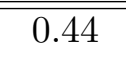 & "0.72 & "0.83 & " 0.71 & 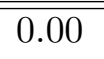 & 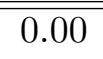 \\
\hline 2 & 20 & 50 & 0.84 & 0.63 & 0.24 & 0.90 & 0.27 & 0.92 & 0.95 & 0.77 & 0.00 & 0.00 \\
\hline 2 & 20 & 100 & 0.96 & 0.85 & 0.44 & 1.00 & 0.39 & 0.99 & 0.99 & 0.97 & 0.00 & 0.00 \\
\hline 2 & 50 & 20 & 0.78 & 0.66 & 0.22 & 0.87 & 0.46 & 0.88 & 0.93 & 0.95 & 0.00 & 0.00 \\
\hline 2 & 50 & 50 & 0.88 & 0.93 & 0.24 & 0.97 & 0.27 & 0.99 & 1.00 & 0.99 & 0.00 & 0.00 \\
\hline 2 & 50 & 100 & 1.00 & 1.00 & 0.45 & 1.00 & 0.35 & 1.00 & 1.00 & 1.00 & 0.00 & 0.00 \\
\hline 2 & 100 & 20 & 0.84 & 0.75 & 0.22 & 0.90 & 0.43 & 0.93 & 0.95 & 0.99 & 0.00 & 0.00 \\
\hline 2 & 100 & 50 & 0.98 & 0.96 & 0.23 & 0.97 & 0.22 & 1.00 & 1.00 & 1.00 & 0.00 & 0.00 \\
\hline 2 & 100 & 100 & 1.00 & 1.00 & 0.43 & 1.00 & 0.31 & 1.00 & 1.00 & 1.00 & 0.00 & 0.00 \\
\hline
\end{tabular}

The data contains 2 common factors. I(O) data generated by a Pesaran DGP. Rejection probabilities are based on 5\% cutoff values from Pesaran (2005), Tables $1 b$ and 3b, 5\% cutoff values of the standard normal distribution, or $5 \%$ Dickey-Fuller critical values for the test statistics as specified in the text. 
Table 16: Unit root test statistics for individual series of real exchange rates.

\begin{tabular}{|c|c|c|c|c|c|c|}
\hline & \multicolumn{2}{|c|}{$A D F_{i}$} & \multicolumn{2}{|c|}{$C A D F_{i}$} & \multicolumn{2}{|c|}{$A D F_{\hat{E}}^{c}$} \\
\hline "Country & 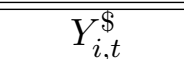 & $\bar{Y}_{i, t}^{D M}$ & 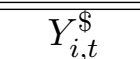 & $\bar{Y}_{i, t}^{D M}$ & 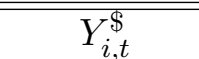 & $\bar{Y}_{i, t}^{D M}$ \\
\hline Austria & -1.4546 & -1.9706 & -1.4507 & -1.7930 & $-2.0148^{* *}$ & -1.0179 \\
\hline Denmark & -1.7667 & -1.8571 & -2.6133 & -2.3326 & $-2.5865^{* *}$ & $-12.3785^{* *}$ \\
\hline Finland & -1.4707 & -1.7878 & -0.8203 & -1.5242 & $-1.7758^{*}$ & $-14.8259^{* *}$ \\
\hline France & -1.3803 & -1.9697 & -2.1514 & $-3.5277^{* *}$ & $-5.3264^{* *}$ & $-7.9788^{* *}$ \\
\hline Germany & -1.3537 & - & -1.6298 & - & $-8.1950^{* *}$ & - \\
\hline Greece & -1.3602 & -2.0247 & -1.2353 & -2.4531 & -0.1947 & -0.5564 \\
\hline Italy & -1.1971 & -1.9952 & -1.5815 & -2.2758 & $-1.9926^{* *}$ & $-3.4528^{* *}$ \\
\hline NL & -1.8189 & -1.1789 & -1.8504 & 0.4805 & $-12.4864^{* *}$ & $-2.4592^{* *}$ \\
\hline Norway & -1.6895 & -1.8398 & -1.4439 & -2.4683 & $-3.2075^{* *}$ & $-2.6420^{* *}$ \\
\hline Portugal & -1.9609 & -1.9522 & -0.6468 & -1.5935 & -1.3352 & $-2.2390^{* *}$ \\
\hline Spain & -1.0189 & -1.9702 & -0.6811 & -1.2196 & $-1.6539^{*}$ & -1.5992 \\
\hline Sweden & -1.3288 & -1.9717 & -2.3255 & -1.5175 & $-13.3224^{* *}$ & $-10.7084^{* *}$ \\
\hline Switzerland & -2.0188 & $-3.0893^{* *}$ & -1.9543 & -2.4054 & $-2.8777^{* *}$ & $-14.4867^{* *}$ \\
\hline UK & $-3.2172^{* *}$ & $-2.8032^{*}$ & -1.7646 & -2.7754 & $-3.7003^{* *}$ & $-12.4749^{* *}$ \\
\hline factor & - & - & - & 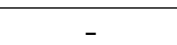 & $-7.6314^{* *}$ & $-7.749^{* *}$ \\
\hline
\end{tabular}

* indicates rejection at $10 \%$ significance level;

** indicates rejection at 5\% significance level.

Table 17: Pooled unit root test statistics panels of real exchange rates.

\begin{tabular}{|l|c|cc|c|}
\hline & Pesaran (2005) & \multicolumn{2}{|c|}{ Moon and Perron (2004) } & Bai and Ng (2004a) \\
\hline \hline & CIPS & $t_{a}^{*}$ & $t_{b}^{*}$ & $P_{\hat{E}}^{c}$ \\
\hline$q_{i, t}^{\$}$ & -1.5821 & -0.0027 & $-1.9418^{* *}$ & $16.6123^{* *}$ \\
$q_{i, t}^{D M}$ & -1.9543 & -0.3720 & $-392.624^{* *}$ & $18.4179^{* *}$ \\
\hline
\end{tabular}

\begin{tabular}{|l|cc|c|}
\hline & \multicolumn{2}{|c|}{ Breitung and Das (2006) } & Sul (2006) \\
\hline \hline & $t_{\text {rob }}$ & $t_{\text {gls }}$ & $t_{\text {fglsrma }}$ \\
\hline$q_{i, t}^{\$}$ & -0.0752 & -1.2160 & 0.5708 \\
$q_{i, t}^{D M}$ & -0.3227 & -0.9335 & $-4.0404^{* *}$ \\
\hline
\end{tabular}

* indicates rejection at $10 \%$ significance level;

** indicates rejection at 5\% significance level. 